February 25, 2014 15:35 WSPC/INSTRUCTION FILE review

Mathematical Models and Methods in Applied Sciences

(c) World Scientific Publishing Company

\title{
New perspectives on polygonal and polyhedral finite element methods
}

\author{
GIANMARCO MANZINI \\ Applied Mathematics and Plasma Physics Group, Theoretical Division, \\ Los Alamos National Laboratory, Los Alamos, NM 87545, USA, gmanzini@lanl.gov \\ Istituto di Matematica Applicata e Tecnologie Informatiche "E. Magenes" CNR, \\ via Ferrata 1, 27100 Pavia, Italy \\ ALESSANDRO RUSSO \\ Dipartimento di Matematica e Applicazioni, Università di Milano-Bicocca, \\ Via Cozzi 53, I-20153, Milano, Italy. alessandro.russo@unimib.it \\ Istituto di Matematica Applicata e Tecnologie Informatiche "E. Magenes" CNR, \\ via Ferrata 1, 27100 Pavia, Italy \\ N. SUKUMAR \\ Department of Civil and Environmental Engineering, University of California, Davis, CA 95616, USA, \\ nsukumar@ucdavis.edu \\ Received (Day Month Year) \\ Revised (Day Month Year) \\ Communicated by (xxxxxxxxx)
}

Generalized barycentric coordinates such as Wachspress and mean value coordinates have been used in polygonal and polyhedral finite element methods. Recently, mimetic finite-difference schemes were cast within a variational framework, and a consistent and stable finite element method on arbitrary polygonal meshes was devised. The method was coined as the Virtual Element Method (VEM), since it did not require the explicit construction of basis functions. This advance provides a more in-depth understanding of mimetic schemes, and also endows polygonal-based Galerkin methods with greater flexibility than three-node and four-node finite element methods. In the VEM, a projection operator is used to realize the decomposition of the stiffness matrix into two terms: a consistent matrix that is known, and a stability matrix that must be positive semi-definite and which is only required to scale like the consistent matrix. In this paper, we first present an overview of previous developments on conforming polygonal and polyhedral finite elements, and then appeal to the exact decomposition in the VEM to obtain a robust and efficient generalized barycentric coordinate-based Galerkin method on polygonal and polyhedral elements. The consistent matrix of the VEM is adopted, and numerical quadrature with generalized barycentric coordinates is used to compute the stability matrix. This facilitates post-processing of field variables and visualization in the VEM, and on the other hand, provides a means to exactly satisfy the patch test with efficient numerical integration in polygonal and polyhedral finite elements. We present numerical examples that demonstrate the sound accuracy and performance of the proposed method. For Poisson problems in $\mathbb{R}^{2}$ and $\mathbb{R}^{3}$, we establish that linearly complete generalized barycentric interpolants deliver optimal rates of convergence in the $L^{2}$ norm and the $H^{1}$ seminorm.

Keywords: Wachspress basis functions, barycentric finite elements, virtual element method, numerical 
integration, consistency

\section{Introduction}

Over the past decade, there has been significant interest in developing non-traditional numerical discretization techniques on polygonal and polyhedral meshes. Among such advances, generalized barycentric coordinates, which are the extension of barycentric coordinates on simplices [1] to polygons and polyhedra, has been a topical research area of focus. Barycentric interpolation is widely used in computer graphics, whereas such interpolating functions have also been adopted as trial and test approximations in finite element methods. Emanating from the seminal work of Wachspress in 1975 [2], the ideas of barycentric coordinates and barycentric interpolation have been extended in recent years to arbitrary polygons in the plane and general polytopes in higher dimensions, which in turn has led to novel solutions in geometry processing (mesh parametrization, image warping, and mesh deformation) and in computational mechanics (material fracture, topology optimization, Stokes flow). In this paper, we present an overview and some new perspectives on the use of generalized barycentric coordinates in Galerkin finite element computations.

On using elements of projective geometry [3], Wachspress [2] constructed a rational basis on convex polygons. Only about a decade ago did renewed interest in Wachspress basis functions arise, for both, graphics [4] and finite element applications [5, 6]. The advent of Floater's mean value coordinate [7] was the turning point in graphics, and now the notion of generalized barycentric coordinates $[8,9,10]$ is well-established. Sibson coordinates [11], Laplace coordinates [12, 13], and discrete harmonic coordinates $[14,15]$ are some of the earliest generalized barycentric coordinates. Since then, many new coordinates have been proposed - metric coordinates [16, 17], harmonic coordinates [18, 19], positive mean value coordinates [20], maximum entropy coordinates [21], complex barycentric coordinates [22, 23], moving least squares coordinates [24], Poisson coordinates [25], and cubic mean value coordinates [26]. Two-dimensional Wachspress coordinates have been extended to convex polytopes [27, 28, 29], and Floater et al. [30] constructed mean value coordinates for polyhedra. Warren [31] proved that rational (Wachspress) barycentric coordinates of degree $n-d$ for a polytope of dimension $d$ bounded by $n$ facets are unique and of minimal degree. The many generalizations, connections, and applications of barycentric coordinates in computer graphics have been extensive [32, 33, 34, 35, 36, 37, 38]. Along with the advances of barycentric coordinates in graphics, there has been a parallel development of barycentric finite element and boundary element methods on polygons and polyhedra $[5,39,40,41,17,42,43,44,45,46,47,48$, $49,50]$, with recent applications in fracture modeling [51, 52, 53], topology optimization $[54,55$, $56]$, mesh generation [57, 58, 59, 60, 61], and error estimates in Sobolev norms [62, 63].

The remainder of this paper is organized as follows. Section 2 introduces the main properties of generalized barycentric coordinates, and in Sections 2.1 and 2.2, the construction of some of the most prominent coordinates on polygons and polyhedra are described. The Galerkin formulation and implementation of polygonal and polyhedral finite elements are discussed in Section 3, with emphasis on connecting the virtual element method $[64,65,66]$ to barycentric finite element methods. We point out the related work of Talischi and Paulino [67] (also in this Special Issue), where the same approach is presented to ameliorate integration errors over polygonal meshes. In Section 4, numerical results for two- and three-dimensional Poisson problems are presented to affirm 
the sound accuracy and optimal rate of convergence of the method. We conclude with some final remarks in Section 5.

\section{Overview of Generalized Barycentric Coordinates}

Barycentric coordinates were first introduced by Möbius as a special kind of homogeneous coordinates with respect to the vertices of a simplex [1]. While unique for simplices, they can be generalized in several ways to arbitrary polygons, polyhedra, higher-dimensional polytopes, and even curves. For planar polygons, generalized barycentric coordinates are readily constructed using many different approaches, such as Wachspress coordinates [2], Sibson coordinates [11, 42], mean value coordinates [7], harmonic coordinates [18], and maximum-entropy coordinates [40].

Let $\Omega_{e} \subset \mathbb{R}^{d}$ be an arbitrary polytope (polygon in $\mathbb{R}^{2}$ or polyhedron in $\mathbb{R}^{3}$ ), with vertices $\mathbf{x}_{1}, \ldots, \mathbf{x}_{n}$. Given weight functions $w_{a}(\mathbf{x}): \Omega_{e} \rightarrow \mathbb{R}$, the functions $\phi_{a}(\mathbf{x}): \Omega_{e} \rightarrow \mathbb{R}(a=1, \ldots, n)$ are called generalized barycentric coordinates (synonymous with shape functions in finite element methods) with respect to $\Omega_{e}$ if they form a partition of unity,

$$
\phi_{a}(\mathbf{x})=\frac{w_{a}(\mathbf{x})}{\sum_{b=1}^{n} w_{b}(\mathbf{x})}, \quad \sum_{a=1}^{n} \phi_{a}(\mathbf{x})=1,
$$

allow us to write any point $\mathbf{x} \in \Omega_{e}$ as an affine combination of the vertices,

$$
\sum_{a=1}^{n} \phi_{a}(\mathbf{x}) \mathbf{x}_{a}=\mathbf{x}
$$

and satisfy the Kronecker-delta property,

$$
\phi_{a}\left(\mathbf{x}_{b}\right)=\delta_{a b} .
$$

Due to the properties in (2.1)-(2.3), these generalized barycentric coordinates can be used to construct trial and test approximations in Galerkin methods. For a scalar-valued function $u(\mathbf{x})$, the interpolant (trial function) $u_{e}^{h}(\mathbf{x}): \Omega_{e} \rightarrow \mathbb{R}$ is written as:

$$
u_{e}^{h}(\mathbf{x})=\sum_{a=1}^{n} \phi_{a}(\mathbf{x}) u_{a}
$$

where due to (2.3), $u_{a}$ assumes the interpretation as the nodal value of the interpolant. Furthermore, many of the applications in computer graphics (mesh deformation, Gourad shading, image warping) and in computational mechanics to solve partial differential equations (satisfaction of the maximum principle, variation-diminishing property, suppressing Runge's phenomenon [68], positive-definite mass matrices in dynamics) greatly benefit from the barycentric coordinates being non-negative,

$$
\phi_{a}(\mathbf{x}) \geq 0,
$$

so that (2.2) becomes a convex combination and $u_{e}^{h}(\mathbf{x})$ is guaranteed to lie inside the convex hull of $\left\{u_{a}\right\}_{a=1}^{n}$. In this study, we adopt generalized barycentric coordinates that satisfy the properties (2.1)-(2.3) and (2.5), and the barycentric interpolant is represented in the form given in (2.4). 


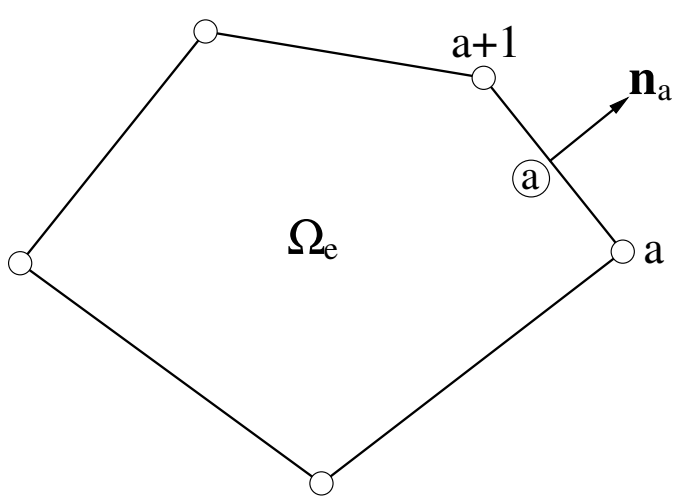

(a)

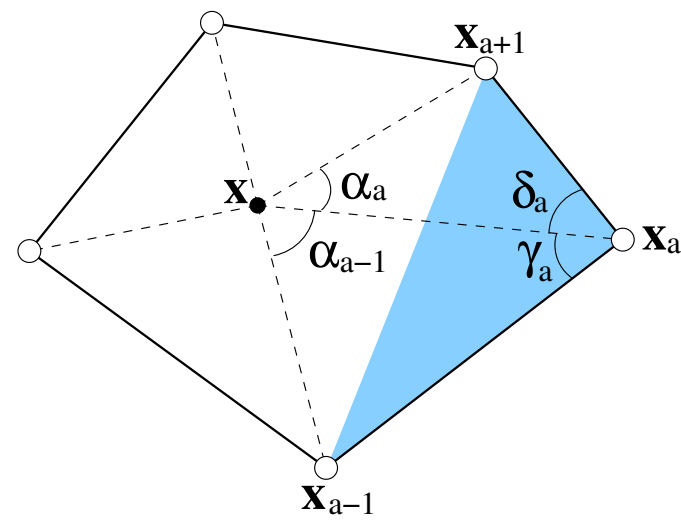

(b)

Fig. 1. Convex polygon. (a) Node numbering in counterclockwise orientation. $e_{a}:=\left(\mathbf{x}_{a}, \mathbf{x}_{a+1}\right)$ is the $a$-th edge, $\mathbf{n}_{a}$ is the unit outward normal to $e_{a}$, and cyclic order is assumed; and (b) Wachspress and mean value coordinates.

\subsection{Barycentric coordinates on polygons}

\subsubsection{Wachspress coordinates}

Wachspress [2] was the first to come up with a generalization of barycentric coordinates to convex polygons for finite element applications. Consider a planar polygonal element $\Omega_{e} \subset \mathbb{R}^{2}$ with $n$ vertices (Fig. 1a). The rational shape functions take the form [2]:

$$
\phi_{a}(\mathbf{x})=\frac{\mathbb{P}_{n-2}(\mathbf{x})}{\mathbb{P}_{n-3}(\mathbf{x})},
$$

where $\mathbb{P}_{k}(\mathbf{x})$ is a bivariate polynomial of degree $k$. For an irregular convex polygon, Wachspress [2] used projective geometry to present the formulation for the shape functions: numerator in the expression for $\phi_{a}(\mathbf{x})$ in (2.6) is proportional to the product of the equations of the edges of the polygon (excluding the two edges that include node $a$ ), whereas the denominator is the degree $n-3$ algebraic curve that passes through the so-called external intersection points (EIPs). The EIPs are the points where the edges intersect; there are $n(n-3) / 2$ EIPs for an $n$-gon, and the algebraic curve that passes through the EIPs is also known as the adjoint of the polygon [69]. Instead of requiring algebraic geometric computations (lines, curves, adjoints of polygons), Meyer et al. [4] proposed an alternative formula for Wachspress's shape functions:

$$
\phi_{a}(\mathbf{x})=\frac{w_{a}(\mathbf{x})}{\sum_{b=1}^{n} w_{b}(\mathbf{x})}, \quad w_{a}(\mathbf{x})=\frac{A\left(\mathbf{x}_{a-1}, \mathbf{x}_{a}, \mathbf{x}_{a+1}\right)}{A\left(\mathbf{x}_{a-1}, \mathbf{x}_{a}, \mathbf{x}\right) A\left(\mathbf{x}_{a}, \mathbf{x}_{a+1}, \mathbf{x}\right)}=\frac{\cot \gamma_{a}+\cot \delta_{a}}{\left\|\mathbf{x}-\mathbf{x}_{a}\right\|^{2}},
$$

where $A(a, b, c)$ is the signed area of triangle $[a, b, c]$ (Fig. 1b), and the last (local) expression in terms of angles is due to Meyer et al. [4].

Recently, simplified formulas were conceived for Wachspress coordinates on polygons and polyhedra [70]. Consider once again the convex polygon $\Omega_{e} \subset \mathbb{R}^{2}$ shown in Fig. 1. Let $\mathbf{n}_{a} \in \mathbb{R}^{2}$ be the unit outward normal to the edge $e_{a}$, with vertices indexed cyclically, i.e., $\mathbf{x}_{n+1}:=\mathbf{x}_{1}$. For any $\mathbf{x}$ in 
$\Omega_{e}$, let $h_{a}(\mathbf{x})$ be the perpendicular distance of $\mathbf{x}$ to the edge $e_{a}$. Then, we can write

$$
h_{a}(\mathbf{x})=\left(\mathbf{x}_{a}-\mathbf{x}\right) \cdot \mathbf{n}_{a}=\left(\mathbf{x}_{a+1}-\mathbf{x}\right) \cdot \mathbf{n}_{a} .
$$

The Wachspress shape functions $\phi_{a}(\mathbf{x}): \Omega_{e} \rightarrow \mathbb{R}(a=1, \ldots, n)$ are defined by the formula [70]

$$
\phi_{a}(\mathbf{x})=\frac{w_{a}(\mathbf{x})}{\sum_{b=1}^{n} w_{b}(\mathbf{x})}, \quad w_{a}(\mathbf{x}):=\frac{\left|\mathbf{n}_{a-1} \times \mathbf{n}_{a}\right|}{h_{a-1}(\mathbf{x}) h_{a}(\mathbf{x})},
$$

where $\times$ denotes the two-dimensional cross product. It is readily shown that the formulas in (2.7) and (2.9) are equivalent. However, (2.7) is in terms of areas of triangles and angles, whereas the formula in (2.9) is much simpler to implement and proves to be more convenient for deriving a bound on $\nabla \phi_{a}[70]$.

\subsubsection{Mean value coordinates}

Floater [7] used the mean value theorem for harmonic functions to construct non-negative linearly precise barycentric coordinates on polygons:

$$
\phi_{a}(\mathbf{x})=\frac{w_{a}(\mathbf{x})}{\sum_{b=1}^{n} w_{b}(\mathbf{x})}, \quad w_{a}(\mathbf{x})=\frac{\tan \left(\alpha_{a-1} / 2\right)+\tan \left(\alpha_{a} / 2\right)}{\left\|\mathbf{x}-\mathbf{x}_{a}\right\|},
$$

where the angle $\alpha_{a}$ is shown in Fig. 1b.

Wachspress coordinates are valid only on convex polygons, whereas mean value coordinates are well-defined for arbitrary planar polygons without self-intersections. Mean value coordinates are positive in the interior of convex polygons. For nonconvex polygons, mean value coordinates are positive in the kernel of the polygon, have a smooth extension outside the polygon, and remain linear on any boundary edge [7,71]; all of these properties are not met by other generalized barycentric coordinates [17]. In barycentric finite element methods, the computation of mean value coordinates and its derivatives are required. To this end, on letting $\mathbf{r}_{a}=\mathbf{x}-\mathbf{x}_{a}\left(r_{a}=\left\|\mathbf{r}_{a}\right\|\right)$, we can write $[71,51]$

$$
\tan \left(\frac{\alpha_{a}}{2}\right)=\frac{\sin \alpha_{a}}{1+\cos \alpha_{i}}=\frac{2 A\left(\mathbf{x}_{a}, \mathbf{x}_{a+1}, \mathbf{x}\right)}{r_{a} r_{a+1}+\mathbf{r}_{a} \cdot \mathbf{r}_{a+1}}=\frac{\left|\mathbf{r}_{a} \times \mathbf{r}_{a+1}\right|}{r_{a} r_{a+1}+\mathbf{r}_{a} \cdot \mathbf{r}_{a+1}},
$$

which is now valid for all points $\mathbf{x}$ that are in the interior of an arbitrary planar (convex and nonconvex) polygon. The denominator vanishes only when $\alpha_{a}=\pi$, i.e., when $\mathbf{x}$ lies on the boundary of the polygon.

\subsubsection{Harmonic and maximum-entropy coordinates}

Functions that satisfy Laplace's equation are known as harmonic functions. With an eye on applications in computer graphics such as character animations, Joshi et al. [18] constructed a new set of generalized barycentric coordinates, which they coined as harmonic coordinates. Harmonic coordinates, $\phi_{a}(\mathbf{x})$, are required to satisfy Laplace's equation with suitable Dirichlet boundary 
conditions prescribed on the boundary of the polytope. For the polygon shown in Fig. 1a, the formulation for harmonic coordinates is [18]:

$$
\begin{aligned}
\nabla^{2} \phi_{a}(\mathbf{x}) & =0 \text { in } \Omega_{e}, \\
\phi_{a}(\mathbf{x}) & =g_{a}(\mathbf{x}) \forall \mathbf{x} \in \partial \Omega_{e},
\end{aligned}
$$

where $g_{a}(\mathbf{x})$ is a piecewise linear function (hat function) on the boundary with $g_{a}\left(\mathbf{x}_{b}\right)=\delta_{a b}$. It is readily shown [18] that the $\phi_{a}(\mathbf{x})$ that solves (2.12) satisfies the conditions (2.1)-(2.3). Since on the boundary, $\phi_{a} \in[0,1]$, then by virtue of the maximum principle for Laplace's equation, $\phi_{a}(\mathbf{x})>0$ in the interior of the polygon. The main drawback of harmonic coordinates is that compared to Wachspress or mean value coordinates, they are relatively expensive to evaluate since they require the solution of the boundary-value problem posed in (2.12).

For a polytope in $\mathbb{R}^{d}$ with $n>d+1$, the constraints in (2.1) and (2.2) do not prescribe unique generalized barycentric coordinates. Motivated by this observation and the fact that a set of shape functions can be viewed as a discrete probability distribution, Sukumar [40] used Jaynes's maximum-entropy principle with Shannon entropy [72] as the objective function to construct polygonal interpolants on convex polygons. On using the relative entropy [73] with the notion of a nodal prior weight function in the variational formulation [74], maximum-entropy coordinates have been extended to arbitrary polygons and polyhedra [21], and also to signed shape functions that possess quadratic precision on planar polygons [75]. Harmonic coordinates [18] and maximum-entropy coordinates [21] are non-negative on arbitrary (convex and nonconvex) polygons and polyhedra.

Now, the essentials of the variational formulation to construct maximum-entropy (max-ent) coordinates on planar polygons are presented. The max-ent variational formulation is: for $\mathbf{x} \in \Omega_{e}$ and given nodal weight functions $w_{a}(\mathbf{x}): \Omega_{e} \rightarrow \mathbb{R}_{+}(a=1, \ldots, n)$, find $\phi(\mathbf{x}): \Omega_{e} \rightarrow \mathbb{R}_{+}^{n}\left(\mathbb{R}_{+}\right.$ is the non-negative orthant) as the solution of the following constrained (concave) optimization problem:

$$
\max _{\phi \in \mathbb{R}_{+}^{n}}\left[-\sum_{a=1}^{n} \phi_{a}(\mathbf{x}) \ln \left(\frac{\phi_{a}(\mathbf{x})}{w_{a}(\mathbf{x})}\right)\right],
$$

subject to the linear constraints in (2.1) and (2.2):

$$
\begin{aligned}
\sum_{a=1}^{n} \phi_{a}(\mathbf{x}) & =1, \\
\sum_{a=1}^{n} \phi_{a}(\mathbf{x}) \mathbf{c}_{a}(\mathbf{x}) & =\mathbf{0},
\end{aligned}
$$

where $\mathbf{c}_{a}=\mathbf{x}_{a}-\mathbf{x}$ are shifted nodal coordinates.

Let $\lambda_{0} \in \mathbb{R}$ and $\boldsymbol{\lambda} \in \mathbb{R}^{2}$ be the Lagrange multipliers associated with the constraints in (2.13b) and $(2.13 \mathrm{c})$, respectively. Then, the Lagrangian is:

$$
\mathcal{L}\left(\boldsymbol{\phi}, \lambda_{0}, \boldsymbol{\lambda}\right)=-\sum_{a=1}^{n} \phi_{a}(\mathbf{x}) \ln \left(\frac{\phi_{a}(\mathbf{x})}{w_{a}(\mathbf{x})}\right)-\lambda_{0}\left(\sum_{a=1}^{n} \phi_{a}(\mathbf{x})-1\right)-\boldsymbol{\lambda} \cdot\left(\sum_{a=1}^{n} \phi_{a}(\mathbf{x}) \mathbf{c}_{a}(\mathbf{x})\right) .
$$


The Karush-Kuhn-Tucker (KKT) first-order optimality condition is:

$$
-1-\ln \phi_{a}(\mathbf{x})+\ln w_{a}(\mathbf{x})-\lambda_{0}-\boldsymbol{\lambda} \cdot \mathbf{c}_{a}(\mathbf{x})=0, \quad(a=1, \ldots, n)
$$

and on setting $\ln Z=1+\lambda_{0}$, where $Z(\mathbf{x}, \boldsymbol{\lambda}): \Omega_{e} \times \mathbb{R}^{2} \rightarrow \mathbb{R}$ is known as the partition function in statistical mechanics, the above yields

$$
\phi_{a}(\mathbf{x})=\frac{w_{a}(\mathbf{x}) \exp \left(-\boldsymbol{\lambda} \cdot \mathbf{c}_{a}(\mathbf{x})\right)}{Z(\mathbf{x}, \boldsymbol{\lambda})}, \quad(a=1, \ldots, n)
$$

and since $\sum_{a=1}^{n} \phi_{a}(\mathbf{x})=1$, therefore

$$
\phi_{a}(\mathbf{x})=\frac{Z_{a}(\mathbf{x}, \boldsymbol{\lambda})}{Z(\mathbf{x}, \boldsymbol{\lambda})}, \quad Z_{a}(\mathbf{x}, \boldsymbol{\lambda}):=w_{a}(\mathbf{x}) \exp \left(-\boldsymbol{\lambda} \cdot \mathbf{c}_{a}(\mathbf{x})\right), \quad Z(\mathbf{x}, \boldsymbol{\lambda}):=\sum_{b=1}^{n} Z_{b}(\mathbf{x}, \boldsymbol{\lambda}) .
$$

Now, we can write the Lagrangian dual function $f: \mathbb{R}^{2} \rightarrow \mathbb{R}$ as

$$
f(\boldsymbol{\lambda})=\max _{\boldsymbol{\phi} \in \mathbb{R}_{+}^{n}} \mathcal{L}(\phi, \boldsymbol{\lambda}),
$$

and on substituting $\mathcal{L}(\cdot)$ from $(2.14)$ and $\phi_{a}(\cdot)$ from (2.15) in the above equation and simplifying, we obtain

$$
f(\boldsymbol{\lambda})=\ln Z(\mathbf{x}, \boldsymbol{\lambda}) .
$$

Hence, the dual problem is:

$$
\boldsymbol{\lambda}^{*}(\mathbf{x})=\underset{\boldsymbol{\lambda} \in \mathbb{R}^{2}}{\operatorname{argmin}} \ln Z(\mathbf{x}, \boldsymbol{\lambda}),
$$

where $\boldsymbol{\lambda}^{*}(\mathbf{x})$ is the converged solution for the Lagrange multiplier vector. The dual problem (unconstrained convex minimization) is efficiently solved using Newton's method [40, 76, 21].

Let $\phi_{a}^{*}(\mathbf{x})$ be the max-ent shape function that corresponds to the converged $\boldsymbol{\lambda}^{*}(\mathbf{x})$, and $\nabla \phi_{a}^{*}(\mathbf{x})$ be the gradient of $\phi_{a}^{*}(\mathbf{x})$. On substituting $\lambda^{*}$ in (2.15), the shape function $\phi_{a}^{*}(\mathbf{x})$ is obtained. The gradient of $\phi_{a}^{*}(\mathbf{x})$ for a constant nodal prior weight function $\left(w_{a}(\mathbf{x})=1\right.$ for all $\left.a\right)$ is [40]:

$$
\boldsymbol{\nabla} \phi_{a}^{*}(\mathbf{x})=\phi_{a}^{*}(\mathbf{x}) \mathbf{c}_{a}(\mathbf{x}) \cdot\left(\mathbf{H}^{*}(\mathbf{x})\right)^{-1}, \quad \mathbf{H}^{*}(\mathbf{x})=\sum_{b=1}^{n} \phi_{b}^{*}(\mathbf{x}) \mathbf{c}_{b}(\mathbf{x}) \otimes \mathbf{c}_{b}(\mathbf{x}),
$$

and the general expression for any $w_{a}(\mathbf{x})$ is given in Reference [77]. Plots of Wachspress and maximum-entropy shape functions on convex polygons are shown in Fig. 2.

\subsubsection{Generalized three-point coordinates}

As the previous sections indicate, there are many variants of linearly precise generalized barycentric coordinates for planar polygons. To better understand this nonuniqeness and to unify some of the different coordinates, Floater et al. [8] provided a generating formula to construct three-point generalized barycentric coordinates $\left(w_{a}(\mathbf{x})\right.$ depends only on $\mathbf{x}_{a-1}, \mathbf{x}_{a}$ and $\left.\mathbf{x}_{a+1}\right)$. If there exist weights $w_{a}(\mathbf{x})>0$ that satisfy the linear precision property in $(2.2)$, then $\phi_{a}(\mathbf{x})>0, \phi_{a}\left(\mathbf{x}_{b}\right)=\delta_{a b}$ and $\phi_{a}(\mathbf{x})$ is piecewise linear on the faces connected to node $a$ [8]. This result also applies to 

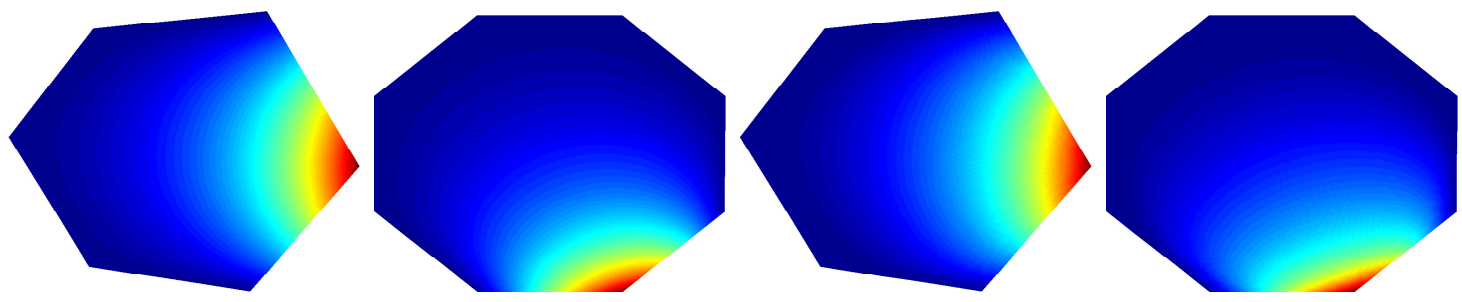

Fig. 2. Shape functions on a hexagon and an octagon.

(a),(b) Wachspress; and (c),(d) Maximum-entropy.
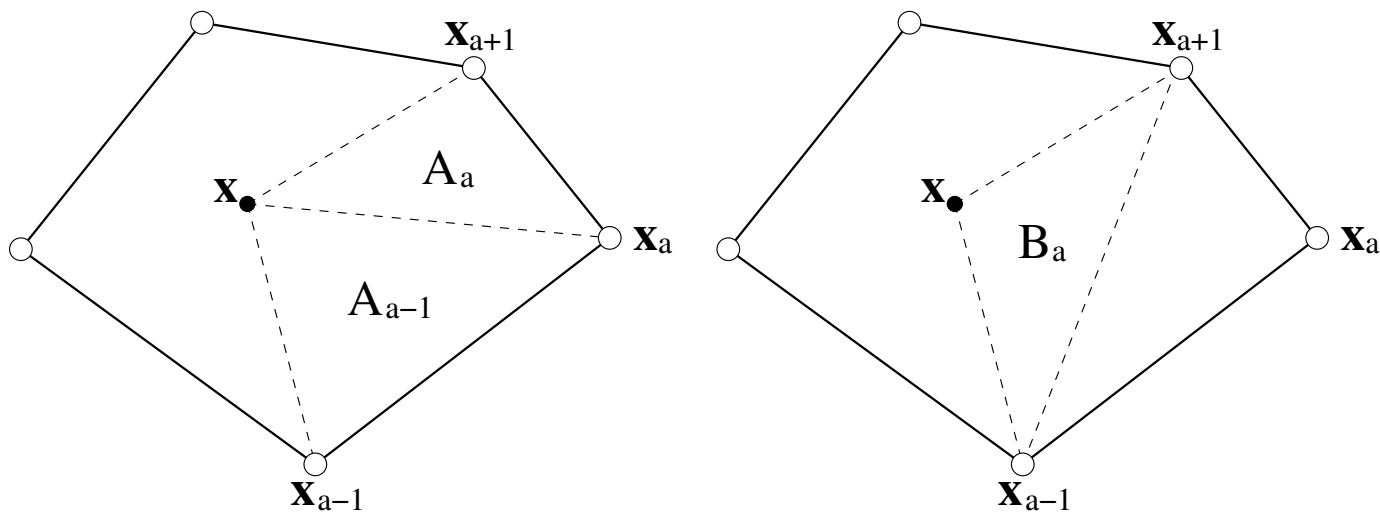

Fig. 3. Three-point generalized barycentric coordinates.

meshfree convex approximation schemes [76, 78]. All three-point barycentric coordinates assume the form $[8]$ :

$$
w_{a}(\mathbf{x})=\frac{c_{a+1}(\mathbf{x}) A_{a-1}(\mathbf{x})-c_{a}(\mathbf{x}) B_{a}(\mathbf{x})+c_{a-1}(\mathbf{x}) A_{a}(\mathbf{x})}{A_{a-1}(\mathbf{x}) A_{a}(\mathbf{x})},
$$

where $c_{a}(\mathbf{x})=f\left(r_{a}\right)$ with $r_{a}=\left\|\mathbf{x}-\mathbf{x}_{a}\right\|$, and $A_{a}$ and $B_{a}$ are illustrated in Fig. 3 . The generating function $f(\mathbf{x}): \mathbb{R}_{+} \rightarrow \mathbb{R}$ exists for all three-point schemes. Floater et al. [8] proved that there exists weights $w_{a}(\mathbf{x})>0$ if and only if $f(r)>0$ (positive), $f^{\prime}(r) \geq 0$ (monotonic), $f^{\prime \prime}(r) \geq 0$ (convex), and $f^{\prime}(r) \leq f(r) / r$ (sublinear); for example, $f(r)=1$ (Wachspress), $f(r)=r$ (mean value coordinates), and $f(r)=r^{2}$ (discrete harmonic coordinates). An example of a five-point generalized barycentric coordinate is the metric coordinates [16].

\subsection{Barycentric coordinates on polyhedra}

For polyhedra, only a few known constructions of generalized barycentric coordinates exist. Warren [27] generalized the original two-dimensional construction by Wachspress to $\mathbb{R}^{d}$, and expressed the barycentric coordinates for the polytope as linear rational combinations of adjoints of dual cones associated with the polytope. On using the notion of the dual polyhedron, Ju et al. [79] presented the barycentric coordinates as the ratio of volumes of certain dual polyhedra. Generalizations of 


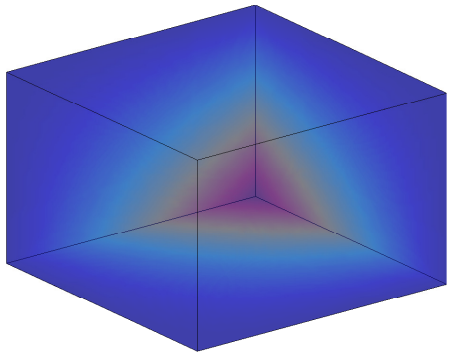

(a)

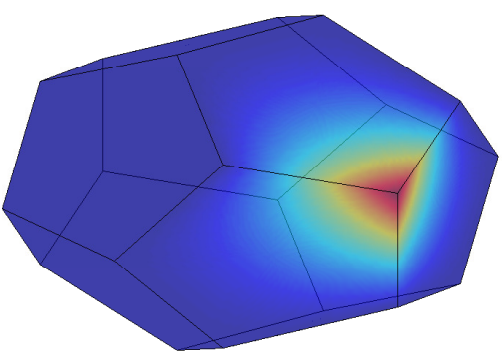

(b)

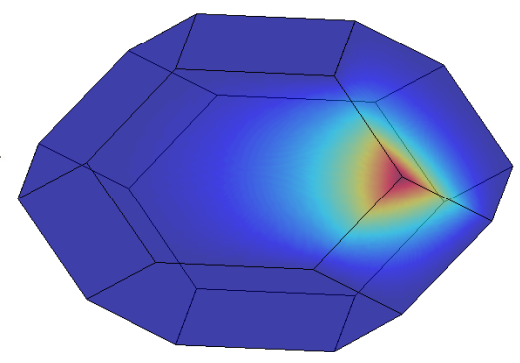

(c)

Fig. 4. Wachspress shape functions on polyhedra. (a) Cube; (b) Dodecahedron; and (c) Tetrakaidecahedron.

mean value coordinates to higher-dimensional polytopes have been realized [30, 80, 81], and they have been used in finite element computations [43, 44]. An extension of metric coordinates [16] to polyhedra is adopted by Kraus and Steinmann [47] for nonlinear continuum computations on polyhedral meshes.

Recently, Floater et al. [70] presented simple formulas and an efficient computer implementation for the generalization of Wachspress coordinates to convex polyhedra proposed by Ju et al. [79]. The algorithm is applicable to polyhedra in $\mathbb{R}^{3}$ that have vertices with valence of three or more. We now present the essential formulas. Let $\Omega_{e} \subset \mathbb{R}^{3}$ be an open, convex polyhedron with vertices $V$, edges $E$, and faces $F$. Let $\mathbf{v}$ be a vertex in $V$. In general there are $k \geq 3$ faces incident on $\mathbf{v}$. Denote these by $\mathbf{f}_{1}, \mathbf{f}_{2}, \ldots, \mathbf{f}_{k}$ in some anticlockwise order as seen from outside $\Omega_{e}$. Let $\mathbf{n}_{1}, \mathbf{n}_{2}, \ldots, \mathbf{n}_{k}$ be the outward unit normals to these faces, respectively. Let $h_{i}(\mathbf{x})>0$ be the perpendicular distance of $\mathbf{x}$ from the face $\mathbf{f}_{i}$, which can be expressed as the scalar product

$$
h_{i}(\mathbf{x})=(\mathbf{v}-\mathbf{x}) \cdot \mathbf{n}_{i} .
$$

The Wachspress shape functions $\phi_{\mathbf{v}}(\mathbf{x}): \Omega_{e} \rightarrow \mathbb{R}(\mathbf{v} \in V)$ are defined by the formula [70]

$$
\phi_{\mathbf{v}}(\mathbf{x})=\frac{w_{\mathbf{v}}(\mathbf{x})}{\sum_{\mathbf{u} \in V} w_{\mathbf{u}}(\mathbf{x})}, \quad w_{\mathbf{v}}(\mathbf{x}):=\frac{1}{h_{1}(\mathbf{x})} \sum_{i=2}^{k-1} \frac{\left(\mathbf{n}_{i} \times \mathbf{n}_{i+1}\right) \cdot \mathbf{n}_{1}}{h_{i}(\mathbf{x}) h_{i+1}(\mathbf{x})} .
$$

The above expression for Wachspress shape functions satisfies the linear precision condition (2.2) [79]. A few plots of Wachspress shape functions on polyhedra are illustrated in Fig. 4.

\section{Polygonal and Polyhedral Finite Elements}

In 1975, Wachspress [2] constructed rational shape functions for polygons, but applications of finite elements on polygons and polyhedra are of more recent origin. Since the early 1990s, many new developments on finite elements on polygons and polyhedra have been conceived. Ghosh and coworkers $[82,83,84]$ introduced a Voronoi-based assumed-stress-hybrid finite element approach 
to model material microstructure and failure, whereas Rashid and coworkers $[85,86,87]$ have advocated the use of nonconforming polygonal and polyhedral shape functions for solid continua. Lin et al. [88, 89, 90] have introduced discontinuous Galerkin formulations on general polygonal and polyehdral meshes. Over the past decade, there has been continued efforts to further the development of polygonal [5, 39, 52, 55] and polyhedral finite element methods [43, 44, 47, 49] using generalized barycentric coordinates.

\subsection{Virtual element method}

Consider the following Poisson boundary-value problem with non-homogeneous Dirichlet boundary conditions:

$$
\begin{aligned}
-\nabla^{2} u & =f \text { in } \Omega \subset \mathbb{R}^{d}, \\
u & =g \text { on } \partial \Omega,
\end{aligned}
$$

whose weak form is: find $u \in H^{1}(\Omega)$ such that

$$
a(u, w)=\ell(w) \quad \forall w \in H_{0}^{1}(\Omega), \quad a(u, w)=\int_{\Omega} \nabla u \cdot \nabla w d \mathbf{x}, \quad \ell(w)=\int_{\Omega} f w d \mathbf{x} .
$$

Mimetic Finite Difference (MFD) schemes are constructed so that fundamental relations of calculus such Green-Gauss formulas (for example, duality, integration by parts, etc.) involving discrete definitions of div, grad, and curl operators on a grid are exactly met [91]. The Support Operator Method (SOM), developed by Shashkov et al. [92], was originally intended to solve linear diffusion problems in mixed form on unstructured quadrilateral and hexahedral meshes [93, 94, 95, 96, 97]. Then, this method evolved into the Mimetic Finite Difference method, which first extended the discretization of differential operators to unstructured meshes with elements of very general shape $[98,99,100,101,102,103]$. Over the past decade, the MFD method has also been successfully employed to solve different partial differential equations. A representative list of problems treated using MFD includes electromagnetism [104, 105, 106, 107, 108], continuum mechanics [109], gas dynamics [110, 111], linear diffusion in primal form [112, 113], convectiondiffusion [114, 115], Stokes flow [116, 117, 118, 119], elasticity [120], Reissner-Mindlin plates [121, 122], eigenvalues [123], and two-phase flows in porous media [124]. A posteriori estimators for the mimetic discretizations appear in References $[125,126]$, and high-order mimetic discretizations are developed in References [127, 128, 129].

Recently, the MFD method has evolved into the virtual element method (VEM), which recasts the mimetic discretization in a variational setting [64]. Herein, we list the most important ingredients of the MFD methods that are inherited by the VEM. In the VEM, an algebraic construction of the stiffness matrix is realized, without the explicit construction of basis functions (basis functions are virtual). The formulation of the VEM as a finite element method of any order of accuracy and regularity on meshes with polygonal and polyhedral cells of very general shape is thus straightforward. In the spirit of the Lax equivalence theorem (consistency + stability $\rightarrow$ convergence) for finite-difference schemes, the construction in the VEM is designed to permit the satisfaction of the consistency and stability conditions. The consistency condition is stated as an exactness property that must be met when the exact solution is a linear polynomial. The patch test is passed on 
convex and nonconvex polygonal and polyhedral meshes, without the use of an explicit basis. Now, we present the essential ideas in the VEM and list the pertinent discrete operators; further details and proofs are available in Reference [64].

Consider a domain $\Omega \subset \mathbb{R}^{d}(d=2,3)$, which is discretized using polygonal (polyhedral) elements in $\mathbb{R}^{2}\left(\mathbb{R}^{3}\right)$ with $\Omega=\cup \Omega_{e}$, where $\Omega_{e}$ is a finite element. A polygon $\Omega_{e}$ with $n$ vertices is shown in Fig. 1a. Let $\mathbf{x} \in \mathbb{R}^{d}$ denote a point in the element, and $\left|\Omega_{e}\right|$ be the measure of the element (area in $\mathbb{R}^{2}$ and volume in $\mathbb{R}^{3}$ ). On each element, we use generalized barycentric coordinates $\left\{\phi_{a}(\mathbf{x})\right\}_{a=1}^{n}$ to form the discrete space: $V_{e}^{h}=\operatorname{span}\left(\left\{\phi_{a}(\mathbf{x})\right\}_{a=1}^{n}\right)$. On choosing the trial function of the form given in (2.4):

$$
u_{e}^{h}(\mathbf{x})=\sum_{b=1}^{n} \phi_{b}(\mathbf{x}) u_{b} \in V_{e}^{h},
$$

where $u_{b}$ are nodal degrees of freedom that are associated with the vertices of the element and testing with the shape functions $\phi_{a}(a=1, \ldots, n)$ in the weak form (3.2) leads to the following linear system in each element $\Omega_{e}$ :

$$
\mathbf{K}_{e} \mathbf{d}_{e}=\mathbf{f}_{e}, \quad \mathbf{K}_{e}^{a b}=a\left(\phi_{a}, \phi_{b}\right)=\int_{\Omega_{e}} \nabla \phi_{a} \cdot \nabla \phi_{b} d \mathbf{x} .
$$

In the virtual element formulation, we restrict our attention to basis functions that span the space of functions of degree 1 (affine functions). Let $\mathcal{P}$ be the vector space that is spanned by affine polynomials, and $\mathbf{m}=\mathbf{x} \equiv\left\{\begin{array}{ll}x y & y\end{array}\right\}^{T}$ and $\mathbf{m}=\mathbf{x} \equiv\left\{\begin{array}{lll}x & y & z\end{array}\right\}^{T}$ be the vector that contains linear monomials in $\mathbb{R}^{2}$ and $\mathbb{R}^{3}$, respectively. We define a projection operator $\Pi: V_{e}^{h} \rightarrow \mathcal{P}\left(\Omega_{e}\right)$ such that it satisfies the following orthogonality condition in the inner product $a(\cdot, \cdot)$ :

$$
a(p, v-\Pi v)=0 \quad \forall p \in \mathcal{P}\left(\Omega_{e}\right), v \in V_{e}^{h},
$$

and since the constant function is in the kernel (null-space) of the projection operator, we need one more equation to determine the constant coefficient of $\Pi v$. To this end, we choose an appropriate projection operator $\Pi_{0}: V_{e}^{h} \rightarrow \mathbb{R}$ onto constants and we require

$$
\Pi_{0}(\Pi v)=\Pi_{0} v
$$

which allows us to fully determine $\Pi v \in \mathcal{P}\left(\Omega_{e}\right)$. The specific choice for the operator $\Pi_{0}$ that we adopt is the following:

$$
\Pi_{0} v=\frac{1}{n} \sum_{a=1}^{n} v\left(\mathbf{x}_{a}\right)
$$

From (3.3), the element stiffness matrix $\mathbf{K}_{e}^{a b}$ is given by

$$
\mathbf{K}_{e}^{a b}=a\left(\phi_{a}, \phi_{b}\right) .
$$

On writing $\phi_{a}=\Pi \phi_{a}+(I-\Pi) \phi_{a}$ and a similar expression for $\phi_{b}$, and substituting in (3.5), we obtain

$$
\mathbf{K}_{e}^{a b}=a\left(\Pi \phi_{a}+(I-\Pi) \phi_{a}, \Pi \phi_{b}+(I-\Pi) \phi_{b}\right),
$$


or

$$
\mathbf{K}_{e}^{a b}=a\left(\Pi \phi_{a}, \Pi \phi_{b}\right)+a\left((I-\Pi) \phi_{a},(I-\Pi) \phi_{b}\right)+a\left((I-\Pi) \phi_{a}, \Pi \phi_{b}\right)+a\left(\Pi \phi_{a},(I-\Pi) \phi_{b}\right) .
$$

But since $\phi_{a}, \phi_{b} \in H^{1}\left(\Omega_{e}\right), \Pi \phi_{a} \in \mathcal{P}\left(\Omega_{e}\right)$ and $\Pi \phi_{b} \in \mathcal{P}\left(\Omega_{e}\right)$, the last two terms vanish in the above equation due to the orthogonality condition (3.4a). Hence, we obtain

$$
\mathbf{K}_{e}^{a b}=a\left(\Pi \phi_{a}, \Pi \phi_{b}\right)+a\left((I-\Pi) \phi_{a},(I-\Pi) \phi_{b}\right) .
$$

Now, we can write $\Pi \phi_{a}=s_{0}^{a}+\sum_{\beta} m_{\beta} s_{\beta}^{a}$, where the vector $\mathbf{s}^{a}$ is obtained from the orthogonality condition (3.4a), while the constant $s_{0}^{a}$ is determined by (3.4b); note that $a\left(m_{\alpha}, s_{0}^{a}\right)=0$ since $s_{0}^{a}$ is a constant. On substituting $v=\phi_{a}, p=m_{\alpha}$ and the above expression for $\Pi \phi_{a}$ in (3.4a), we obtain

$$
a\left(m_{\alpha}, \Pi \phi_{a}\right)=a\left(m_{\alpha}, \phi_{a}\right) \Rightarrow \sum_{\beta=1}^{d} a\left(m_{\alpha}, m_{\beta}\right) s_{\beta}^{a}=a\left(m_{\alpha}, \phi_{a}\right)
$$

for $\alpha=1, \ldots, d$, which leads to the linear system of equations:

$$
\mathbf{G s}^{a}=\mathbf{g}^{a}, \quad \mathbf{G}_{\alpha \beta}=a\left(m_{\alpha}, m_{\beta}\right), \quad \mathbf{g}_{\alpha}^{a}=a\left(m_{\alpha}, \phi_{a}\right), \quad \mathbf{s}^{a}=\left\{s_{1}^{a} \ldots s_{d}^{a}\right\}^{T} .
$$

Note that $\mathbf{G}=\int_{\Omega_{e}} \nabla \mathbf{m} \cdot \nabla \mathbf{m} d \mathbf{x}=\int_{\Omega_{e}} \nabla \mathbf{x} \cdot \nabla \mathbf{x} d \mathbf{x}=\left|\Omega_{e}\right| \mathbf{I}$. The solution of (3.7) is:

$$
\begin{aligned}
& \mathbf{s}^{a}=\mathbf{G}^{-1} \mathbf{g}^{a}, \\
& \mathbf{g}^{a}=a\left(\mathbf{m}, \phi_{a}\right)=\int_{\Omega_{e}} \nabla \mathbf{m} \cdot \nabla \phi_{a} d \mathbf{x}=\int_{\Omega_{e}} \mathbf{I} \cdot \nabla \phi_{a} d \mathbf{x}=\int_{\Omega_{e}} \nabla \phi_{a} d \mathbf{x}=\int_{\partial \Omega_{e}} \phi_{a} \mathbf{n} d S,
\end{aligned}
$$

where the divergence theorem ( $\mathbf{n}$ is the unit outward normal on the boundary) and the fact that $\nabla^{2} m_{\alpha}=0$ are used. Since $\phi_{a}$ is known on the boundary of the element, $\mathbf{g}^{a}$ is readily evaluated. Let us define the matrices $\mathbf{R}$ and $\mathbf{N}$ as follows:

$$
\mathbf{R}=\frac{1}{2}\left[\begin{array}{c}
\ell_{n} \mathbf{n}_{n}+\ell_{1} \mathbf{n}_{1} \\
\ell_{1} \mathbf{n}_{1}+\ell_{2} \mathbf{n}_{2} \\
\cdots \\
\ell_{n-1} \mathbf{n}_{n-1}+\ell_{n} \mathbf{n}_{n}
\end{array}\right], \quad \mathbf{N}=\left[\begin{array}{cc}
x_{1} & y_{1} \\
x_{2} & y_{2} \\
\cdots & \cdots \\
x_{n} & y_{n}
\end{array}\right]
$$

for polygons, where $\ell_{a}(a=1, \ldots, n)$ is the length of edge $a$, and the $a$-th row of $\mathbf{R}$ is $\left(\mathbf{g}^{a}\right)^{T}$. For a polyhedral element,

$$
\mathbf{R}=\left[\begin{array}{c}
w_{1}^{1}\left|f_{1}^{1}\right| \mathbf{n}_{1}^{1}+\ldots+w_{1}^{k_{1}}\left|f_{1}^{k_{1}}\right| \mathbf{n}_{1}^{k_{1}} \\
w_{2}^{1}\left|f_{2}^{1}\right| \mathbf{n}_{2}^{1}+\ldots+w_{2}^{k_{2}}\left|f_{2}^{k_{2}}\right| \mathbf{n}_{2}^{k_{2}} \\
\ldots \\
w_{n}^{1}\left|f_{n}^{1}\right| \mathbf{n}_{n}^{1}+\ldots+w_{n}^{k_{n}}\left|f_{n}^{k_{n}}\right| \mathbf{n}_{n}^{k_{n}}
\end{array}\right], \quad \mathbf{N}=\left[\begin{array}{ccc}
x_{1} & y_{1} & z_{1} \\
x_{2} & y_{2} & z_{2} \\
\ldots \ldots & \ldots \\
x_{n} & y_{n} & z_{n}
\end{array}\right]
$$

where $k_{a}$ are the number of faces that are incident to node $a,\left|f_{a}^{k}\right|$ is the measure (area) of the $k$-th face incident to node $a, \mathbf{n}_{a}^{k}$ is the unit outward normal to the $k$-th face that is incident to node $a$, and $w_{a}^{k}$ is the corresponding quadrature weight, which is obtained from a linearly precise integration rule. The choice of these weights is not unique as, in principle, any set of weights associated with the polyhedral vertices that provides a second-order accurate quadrature can be used. In Appendix A, we show the existence of such weights in a constructive way that yields a formula for their calculation. Their non-uniqueness is also discussed. 
From (3.8) and (3.9), we obtain

$$
\mathbf{S}=\left\{\mathbf{s}^{1} \mathbf{s}^{2} \ldots \mathbf{s}^{n}\right\}=\mathbf{G}^{-1}\left\{\mathbf{g}^{1} \mathbf{g}^{2} \ldots \mathbf{g}^{n}\right\}=\mathbf{G}^{-1} \mathbf{R}^{T},
$$

and we can express the first term in (3.6) as

$$
a\left(\Pi \phi_{a}, \Pi \phi_{b}\right)=\sum_{\alpha} \sum_{\beta} s_{\alpha}^{a} a\left(m_{\alpha}, m_{\beta}\right) s_{\beta}^{b}=\mathbf{S}^{T} \mathbf{G} \mathbf{S}=\frac{\mathbf{R R}^{T}}{\left|\Omega_{e}\right|},
$$

where (3.10) and $\mathbf{G}^{-1}=\mathbf{I} /\left|\Omega_{e}\right|$ have been used. The matrix $\mathbf{G}$ can also be represented as:

$$
\mathbf{G}=a(\mathbf{m}, \mathbf{m})=\int_{\Omega_{e}} \nabla \mathbf{x} \cdot \nabla \mathbf{x} d \mathbf{x}=\int_{\partial \Omega_{e}} \mathbf{x} \otimes \mathbf{n} d S=\sum_{a} \mathbf{x}_{a} \otimes \int_{\partial \Omega_{e}} \phi_{a} \mathbf{n} d S=\mathbf{N}^{T} \mathbf{R},
$$

where the divergence theorem has been invoked. Since $\mathbf{G}$ is a symmetric matrix, it follows that $\mathbf{N}^{T} \mathbf{R}$ is also a symmetric matrix, i.e., $\mathbf{G}=\mathbf{N}^{T} \mathbf{R}=\mathbf{R}^{T} \mathbf{N}$.

Let us now derive the matrix representation of the projection operator $\Pi$. We recall that

$$
\Pi \phi_{a}(\mathbf{x})=s_{0}^{a}+(\mathbf{m}(\mathbf{x}))^{T} \mathbf{s}^{a},
$$

and on collecting terms for all $a$ in a row vector, we have

$$
\begin{aligned}
\left\{\Pi \phi_{1}(\mathbf{x}) \Pi \phi_{2}(\mathbf{x}) \ldots \Pi \phi_{n}(\mathbf{x})\right\} & =\left\{s_{0}^{1} s_{0}^{2} \ldots s_{0}^{n}\right\}+(\mathbf{m}(\mathbf{x}))^{T}\left\{\mathbf{s}^{1} \mathbf{s}^{2} \ldots \mathbf{s}^{n}\right\} \\
& =\mathbf{S}_{0}+(\mathbf{m}(\mathbf{x}))^{T} \mathbf{S}=\mathbf{S}_{0}+(\mathbf{m}(\mathbf{x}))^{T} \mathbf{G}^{-1} \mathbf{R}^{T},
\end{aligned}
$$

where (3.10) has been used. On using the linear precision condition (2.2), we can write

$$
\mathbf{m}(\mathbf{x})=\mathbf{x}=\sum_{a=1}^{n} \phi_{a}(\mathbf{x}) \mathbf{x}_{a}=\mathbf{N}^{T}\left\{\begin{array}{c}
\phi_{1}(\mathbf{x}) \\
\phi_{2}(\mathbf{x}) \\
\ldots \\
\phi_{n}(\mathbf{x})
\end{array}\right\}
$$

Substituting the above expression for $\mathbf{m}(\mathbf{x})$ in (3.14) yields:

$$
\left\{\Pi \phi_{1}(\mathbf{x}) \Pi \phi_{2}(\mathbf{x}) \ldots \Pi \phi_{n}(\mathbf{x})\right\}=\mathbf{S}_{0}+\left\{\phi_{1}(\mathbf{x}) \phi_{2}(\mathbf{x}) \ldots \phi_{n}(\mathbf{x})\right\} \mathbf{N G}^{-1} \mathbf{R}^{T} .
$$

Let $\tilde{\Pi}$ be the projection onto linear functions, i.e., $\tilde{\Pi} \phi_{a}(\mathbf{x})=(\mathbf{m}(\mathbf{x}))^{T} \mathbf{s}^{a}$. Then, $\Pi \phi_{a}(\mathbf{x})$ admits the following direct decomposition:

$$
\Pi \phi_{a}(\mathbf{x})=\Pi_{0} \phi_{a}(\mathbf{x})+\left(I-\Pi_{0}\right) \tilde{\Pi} \phi_{a}(\mathbf{x}),
$$

which on rearranging yields

$$
\Pi \phi_{a}(\mathbf{x})=\tilde{\Pi} \phi_{a}(\mathbf{x})+\Pi_{0}(I-\tilde{\Pi}) \phi_{a}(\mathbf{x}) .
$$

We point out that the above equation can be readily derived by applying $\Pi_{0}$ to (3.13), and noting that $\Pi_{0} s_{0}^{a}=s_{0}^{a}$ and $\Pi_{0}\left(\Pi \phi_{a}(\mathbf{x})\right)=\Pi_{0} \phi_{a}(\mathbf{x})$ from $(3.4 \mathrm{~b})$. Hence, the matrix representation of the three projection operators must satisfy the following relation:

$$
\boldsymbol{\Pi}=\tilde{\boldsymbol{\Pi}}+\boldsymbol{\Pi}_{0}(\mathbf{I}-\tilde{\boldsymbol{\Pi}}) .
$$

From (3.4c), we obtain the following expression for $\boldsymbol{\Pi}_{0}$ :

$$
\boldsymbol{\Pi}_{0}=\frac{\mathbb{1}^{T}}{n}, \quad \mathbb{1}=\left\{\begin{array}{lll}
1 & 1 \ldots 1
\end{array}\right\}^{T}
$$


is a $n$-dimensional column vector of ones. The matrix $\tilde{\boldsymbol{\Pi}}$ is such that

$$
\left\{\Pi \phi_{1}(\mathbf{x}) \Pi \phi_{2}(\mathbf{x}) \ldots \Pi \phi_{n}(\mathbf{x})\right\}=\mathbf{S}_{0}+\left\{\phi_{1}(\mathbf{x}) \phi_{2}(\mathbf{x}) \ldots \phi_{n}(\mathbf{x})\right\} \tilde{\boldsymbol{\Pi}},
$$

and on comparing (3.15) and (3.19), we have

$$
\tilde{\mathbf{\Pi}}=\mathbf{N G}^{-1} \mathbf{R}^{T}=\frac{\mathbf{N R}^{T}}{\left|\Omega_{e}\right|},
$$

where the projection matrix $\tilde{\boldsymbol{\Pi}}$ has the interpretation of an oblique projection [130], such that the column vectors of $\mathbf{N}$ form a basis for the range of the projection. Since the columns of $\mathbf{R}$ form a basis for the orthogonal complement of the kernel of the projection, i.e., $\operatorname{img}(\mathbf{R})=(\operatorname{ker}(\boldsymbol{\Pi}))^{\perp}$, then $\tilde{\boldsymbol{\Pi}}=\mathbf{N}\left(\mathbf{R}^{T} \mathbf{N}\right)^{-1} \mathbf{R}^{T}=\mathbf{N} \mathbf{R}^{T} /\left|\Omega_{e}\right|$, since $\mathbf{R}^{T} \mathbf{N}=\mathbf{G}=\left|\Omega_{e}\right| \mathbf{I}$ from (3.12).

Now, from (3.6) and (3.11), the decomposition for the stiffness matrix in the virtual element method is written as

$$
\mathbf{K}_{e}=\boldsymbol{\Pi}^{T} \mathbf{K}_{e} \boldsymbol{\Pi}+(\mathbf{I}-\boldsymbol{\Pi})^{T} \mathbf{K}_{e}(\mathbf{I}-\boldsymbol{\Pi})=\frac{\mathbf{R R}^{T}}{\left|\Omega_{e}\right|}+(\mathbf{I}-\mathbf{\Pi})^{T} \mathbf{K}_{e}(\mathbf{I}-\mathbf{\Pi}),
$$

where $\boldsymbol{\Pi}$ is defined in (3.17). In the VEM, the first term in (3.21) provides consistency and the second term lends stability. It has been shown in Reference [64] that $\mathbf{K}_{e}$ on the right-hand-side can be replaced by the identity matrix, still yielding a convergent scheme:

$$
\mathbf{K}_{e}=\frac{\mathbf{R R}^{T}}{\left|\Omega_{e}\right|}+(\mathbf{I}-\boldsymbol{\Pi})^{T}(\mathbf{I}-\mathbf{\Pi}) .
$$

\subsection{Algebraic form of the consistency condition}

The exact solution for the patch test is an affine function: $u(\mathbf{x})=\alpha+\boldsymbol{\beta} \cdot \mathbf{x}$. Now, let the nodal value $d_{a}$ stem from this affine field: $d_{a}=\alpha+\boldsymbol{\beta} \cdot \mathbf{x}_{a}$. From the definition of matrix $\mathbf{K}_{e}$ and using (2.1) and (2.2), it follows that:

$$
\begin{aligned}
\sum_{b=1}^{n} \mathbf{K}_{e}^{a b}\left(\alpha+\boldsymbol{\beta} \cdot \mathbf{x}_{b}\right) & =\sum_{b=1}^{n} \int_{\Omega_{e}} \nabla \phi_{a} \cdot \nabla\left(\phi_{b}\left(\alpha+\boldsymbol{\beta} \cdot \mathbf{x}_{b}\right)\right) d \mathbf{x} \\
& =\alpha \int_{\Omega_{e}} \nabla \phi_{a} \cdot \nabla\left(\sum_{b=1}^{n} \phi_{b}\right)+\int_{\Omega_{e}} \nabla \phi_{a} \cdot \nabla\left(\boldsymbol{\beta} \cdot \sum_{b=1}^{n} \phi_{b} \mathbf{x}_{b}\right) d \mathbf{x} \\
& =\alpha \int_{\Omega_{e}} \nabla \phi_{a} \cdot \nabla(1)+\int_{\Omega_{e}} \nabla \phi_{a} \cdot \nabla(\boldsymbol{\beta} \cdot \mathbf{x}) d \mathbf{x} \\
& =\alpha \cdot 0+\boldsymbol{\beta} \cdot \int_{\Omega_{e}} \nabla \phi_{a} d \mathbf{x}=\alpha \cdot 0+\boldsymbol{\beta} \cdot \int_{\partial \Omega_{e}} \phi_{a} \mathbf{n} d S \quad(a=1, \ldots, n) .
\end{aligned}
$$

Equating the coefficients of $\alpha$ and $\boldsymbol{\beta}$ on the left-most and the right-most sides, we obtain

$$
\begin{aligned}
\sum_{b=1}^{n} \mathbf{K}_{e}^{a b} & =0, \\
\sum_{b=1}^{n} \mathbf{K}_{e}^{a b} \mathbf{x}_{b} & =\int_{\partial \Omega_{e}} \phi_{a} \mathbf{n} d S \quad(a=1, \ldots, n),
\end{aligned}
$$


which must be satisfied to ensure that the polygonal or polyhedral finite element solution reproduces an affine function. The condition (3.23a) indicates that the constant vector must lie in the kernel of $\mathbf{K}_{e}$. Furthermore, since the nodal coordinates $\mathbf{x}_{b}$ on the left-hand side of (3.23b) form the columns of matrix $\mathbf{N}$ and the right-hand side in (3.23b) is just $\mathbf{R}$ in (3.9), we find that $\mathbf{K}_{e}$ must satisfy

$$
\mathbf{K}_{e} \mathbf{N}=\mathbf{R},
$$

which is the linear consistency condition in mimetic finite difference schemes.

\subsection{Combined finite element and virtual element approach}

As in meshfree methods [131], since non-polynomial shape functions are used in polygonal and polyhedral finite element methods (PFEM), accurate and efficient numerical integration is a pertinent issue in these methods. In polygonal finite element computations, on partitioning each $n$-gon into $n$ triangles, and using standard polynomial-precise quadrature rules in each triangle, the patch test is only met to $10^{-4}-10^{-8}$ accuracy $[39,132]$. With an eye on satisfying the patch test and requiring fewer integration points to compute the stiffness matrix, nodally integrated meshfree methods that use a smoothed strain operator $[133,134]$ have been proposed. This modified integration rule is also used in the smoothed finite element method $[135,136]$, and a variant of the same is adopted by Bishop [49]. Herein, we appeal to the virtual element method [64] (see previous section) that has firm mathematical underpinnings, to ensure consistency (patch test) and stability in generalized barycentric coordinates-based polygonal and polyhedral finite element methods.

From (3.21), we can write the relation for the exact decomposition of the stiffness matrix as

$$
\mathbf{K}_{e}=\frac{\mathbf{R R}^{T}}{A_{e}}+(\mathbf{I}-\mathbf{\Pi})^{T} \mathbf{K}_{e}(\mathbf{I}-\mathbf{\Pi}),
$$

and now we replace $\mathbf{K}_{e}$ on the right-hand side by $\mathbf{K}_{e}^{t}$, which is an approximation for the element stiffness matrix that is evaluated using numerical quadrature. Such a choice retains consistency and stability of the method. To compute $\mathbf{K}_{e}^{t}$, we use a $t$-th order polynomial-precise quadrature rule on each simplex-partition of the polygon or polyhedron. For ease of implementation, we use $\tilde{\boldsymbol{\Pi}}$ instead of $\boldsymbol{\Pi}$ in (3.25), which is justified by virtue of the following proposition.

Proposition 3.1. If the constant vector lies in the kernel of matrix $\mathbf{K}_{e}^{t}\left(\mathbf{K}_{e}^{t} \mathbb{1}=\mathbf{0}\right)$, then the stabilization term satisfies the relation

$$
(\mathbf{I}-\mathbf{\Pi})^{T} \mathbf{K}_{e}^{t}(\mathbf{I}-\mathbf{\Pi})=(\mathbf{I}-\tilde{\mathbf{\Pi}})^{T} \mathbf{K}_{e}^{t}(\mathbf{I}-\tilde{\mathbf{\Pi}}) .
$$

Proof. On using (3.17), we can write

$$
(\mathbf{I}-\mathbf{\Pi})^{T} \mathbf{K}_{e}^{t}(\mathbf{I}-\boldsymbol{\Pi})=\left(\mathbf{I}-\tilde{\mathbf{\Pi}}-\boldsymbol{\Pi}_{0}(\mathbf{I}-\tilde{\mathbf{\Pi}})\right)^{T} \mathbf{K}_{e}^{t}\left(\mathbf{I}-\tilde{\mathbf{\Pi}}-\boldsymbol{\Pi}_{0}(\mathbf{I}-\tilde{\mathbf{\Pi}})\right),
$$

and since $\boldsymbol{\Pi}_{0}=\mathbb{1}^{T} / n$ from (3.18), therefore $\mathbf{K}_{e}^{t} \boldsymbol{\Pi}_{0}=\boldsymbol{\Pi}_{0} \mathbf{K}_{e}^{t}=\mathbf{0}$, and the above relation simplifies to

$$
(\mathbf{I}-\mathbf{\Pi})^{T} \mathbf{K}_{e}^{t}(\mathbf{I}-\boldsymbol{\Pi})=\left(\mathbf{I}-\tilde{\mathbf{\Pi}}-\boldsymbol{\Pi}_{0}(\mathbf{I}-\tilde{\mathbf{\Pi}})\right)^{T} \mathbf{K}_{e}^{t}(\mathbf{I}-\tilde{\mathbf{\Pi}})=(\mathbf{I}-\tilde{\mathbf{\Pi}})^{T} \mathbf{K}_{e}^{t}(\mathbf{I}-\tilde{\mathbf{\Pi}}),
$$

which is the desired result. 
Using Proposition 3.1, $\mathbf{K}_{e}$ is computed via the following expression in the combined method (coined as PFEM-VEM):

$$
\mathbf{K}_{e}=\frac{\mathbf{R R}^{T}}{A_{e}}+(\mathbf{I}-\tilde{\mathbf{\Pi}})^{T} \mathbf{K}_{e}^{t}(\mathbf{I}-\tilde{\mathbf{\Pi}})
$$

where $\tilde{\boldsymbol{\Pi}}$ is given in (3.20).

Finally, we have the following result on the exactness of $\mathbf{K}_{e}$ for VEM, PFEM, and PFEM-VEM.

Proposition 3.2. Let $p$ and $q$ be two linear polynomials defined on e. We denote their degrees of freedom by $\mathbf{p}=\left(p_{a}\right), \mathbf{q}=\left(q_{a}\right)$, so that $p(\mathbf{x})=\sum_{a} p_{a} \phi_{a}(\mathbf{x})$ holds, and a similar relation applies for $q$ with $q_{a}$ instead of $p_{a}$. Then, it follows that

$$
\mathbf{p}^{T} \mathbf{K}_{e} \mathbf{q}=\int_{\Omega_{e}} \nabla p \cdot \nabla q d \mathbf{x}=\left|\Omega_{e}\right| \nabla p \cdot \nabla q,
$$

where $\mathbf{K}_{e}$ can be the PFEM stiffness matrix (3.3), the VEM stiffness matrix (3.22), or the PFEMVEM stiffness matrix (3.26).

Proof. The local stiffness matrix of the VEM is given by (3.6), which can be rewritten as the sum of two integrals:

$$
\mathbf{K}_{e}^{a b}=\int_{\Omega_{e}} \nabla \Pi \phi_{a} \cdot \nabla \Pi \phi_{b} d \mathbf{x}+\int_{\Omega_{e}} \nabla(I-\Pi) \phi_{a} \cdot \nabla(I-\Pi) \phi_{b} d \mathbf{x} .
$$

On noting that $(I-\Pi) p=(I-\Pi) q=0$ since $p$ and $q$ are linear polynomials, we can write

$$
\begin{aligned}
\mathbf{p}^{T} \mathbf{K}_{e} \mathbf{q} & =\sum_{a b} p_{a} q_{b}\left(\int_{\Omega_{e}} \nabla \Pi \phi_{a} \cdot \nabla \Pi \phi_{b} d \mathbf{x}+\int_{\Omega_{e}} \nabla(I-\Pi) \phi_{a} \cdot \nabla(I-\Pi) \phi_{b} d \mathbf{x}\right) \\
& =\int_{\Omega_{e}} \nabla \sum_{a} p_{a} \Pi \phi_{a} \cdot \nabla \sum_{b} p_{b} \Pi \phi_{b} d \mathbf{x}+\int_{\Omega_{e}} \nabla \sum_{a} p_{a}(I-\Pi) \phi_{a} \cdot \nabla \sum_{b} p_{b}(I-\Pi) \phi_{b} d \mathbf{x} \\
& =\int_{\Omega_{e}} \nabla p \phi_{a} \cdot \nabla p_{b} d \mathbf{x}=\left|\Omega_{e}\right| \nabla p \cdot \nabla q .
\end{aligned}
$$

The correction term in the calculation of $\mathbf{p}^{T} \mathbf{K}_{e} \mathbf{q}$ does not play any role since $(I-\Pi) p=(I-\Pi) q=0$ for any pair of linear polynomials $p$ and $q$. Thus, the same result holds for the alternative forms of the stiffness matrix given by (3.25) and (3.26).

Now, let us consider the PFEM formulation, which approximates the stiffness matrix as follows:

$$
\mathbf{K}_{e}^{a b}=\int_{\Omega_{e}} \nabla \phi_{a} \cdot \nabla \phi_{b} d \mathbf{x}=\sum_{t} w_{t} \nabla \phi_{a}\left(\mathbf{x}_{t}\right) \cdot \nabla \phi_{b}\left(\mathbf{x}_{t}\right)+\left|\Omega_{e}\right| \mathcal{O}\left(h^{r}\right),
$$

where $r \geq 1$ is the accuracy order of the quadrature rule $\left\{\left(w_{t}, \mathbf{x}_{t}\right)\right\}$ on $\Omega_{e}$ (we assume that 
$\left.\sum_{t}=w_{t}=\left|\Omega_{e}\right|\right)$. After rearranging the summations in (3.29), we have

$$
\begin{aligned}
\mathbf{p}^{T} \mathbf{K}_{e} \mathbf{q} & =\sum_{a b} p_{a} q_{b} \int_{\Omega_{e}} \nabla \phi_{a} \cdot \nabla \phi_{b} d \mathbf{x} \\
& =\sum_{a b} p_{a} q_{b}\left(\sum_{t} w_{t} \nabla \phi_{a}\left(\mathbf{x}_{t}\right) \cdot \nabla \phi_{b}\left(\mathbf{x}_{t}\right)+\left|\Omega_{e}\right| \mathcal{O}\left(h^{r}\right)\right) \\
& =\sum_{t} w_{t} \nabla \sum_{a} p_{a} \phi_{a}\left(\mathbf{x}_{t}\right) \cdot \nabla \sum_{b} q_{b} \phi_{b}\left(\mathbf{x}_{b}\right)+\left|\Omega_{e}\right| \mathcal{O}\left(h^{r}\right) .
\end{aligned}
$$

Since $r \geq 1$, the quadrature rule is exact for linear polynomials and the approximation error is zero; furthermore, the gradients are constant vectors and the summation of the weights $w_{t}$ returns the measure of $\Omega_{e}$. Therefore, we obtain the desired result:

$$
\mathbf{p}^{T} \mathbf{K}_{e} \mathbf{q}=\sum_{t} w_{t} \nabla p\left(\mathbf{x}_{t}\right) \cdot \nabla q\left(\mathbf{x}_{b}\right)=\left(\sum_{t} w_{t}\right) \nabla p \cdot \nabla q=\left|\Omega_{e}\right| \nabla p \cdot \nabla q .
$$

The condition (3.27) clarifies why the property $\mathbf{N}^{T} \mathbf{K}_{e} \mathbf{N}=\mathbf{N}^{T} \mathbf{R}$ is satisfied to machine precision by all the methods.

\section{Numerical Examples}

We present two- and three-dimensional finite element solutions of the Poisson equation on polygonal and polyhedral meshes, respectively. The numerical integration scheme described in Section 3.3 is used in the computations. First, the patch test is considered and then the rates of convergence in the $L^{2}$ norm and the $H^{1}$ seminorm are presented for a Poisson problem.

\subsection{Two-dimensional Poisson problems}

\subsubsection{Patch test}

For the patch test, we consider the Laplace equation in a unit square with linear Dirichlet boundary conditions. We choose $f(\mathbf{x})=0$ and $g(\mathbf{x})=1-2 x-3 y$ in (3.1). The exact solution is: $u(\mathbf{x})=g(\mathbf{x})$. We consider the VEM, the PFEM formulation using Wachspress shape functions, and the combined PFEM-VEM formulation of Section 3.3. Numerical integration in PFEM is directly performed by partitioning each physical polygon into $n$ subtriangles, and using one-point integration rule in each triangle. For the PFEM-VEM computations, the PFEM stiffness matrix $\mathbf{K}_{e}^{t}(t=1)$ is used in (3.26). For each mesh, we measure the approximation errors using the $L^{2}$ norm and the $H^{1}$ seminorm, and the consistency errors $\|\mathbf{E}\|_{\mathcal{F}}$ and $\left\|\mathbf{N}^{T} \mathbf{E}\right\|_{\mathcal{F}}$, where $\mathbf{E}=\mathbf{K}_{e} \mathbf{N}-\mathbf{R}$. Consistency errors for matrices are defined through the matrix Frobenius norm $\|\cdot\|_{\mathcal{F}}$.

First, we consider the coarsest mesh in a sequence of eight different types of meshes (Fig. 5). Numerical results for the relative error norms and the consistency errors are tabulated in Tables 1 and 2, respectively. Then, we consider the five meshes shown in Fig. 6, which are refinements of the mesh illustrated in Fig. 5d. The corresponding relative error norms and consistency errors on these meshes is listed in Tables 3 and 4. From the above tabulated results, we can infer the following: 


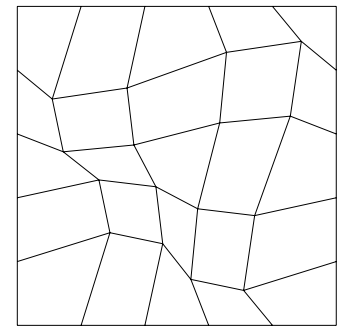

(a)

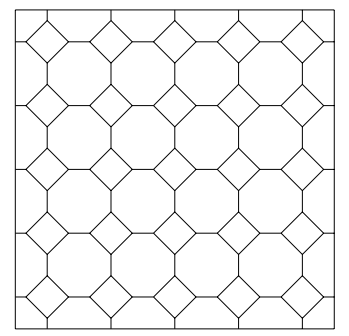

(e)

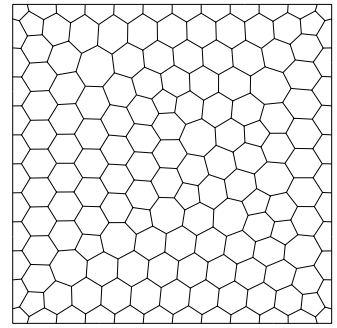

(b)

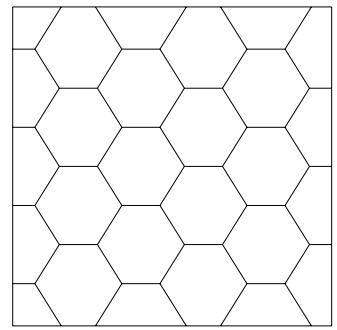

(f)

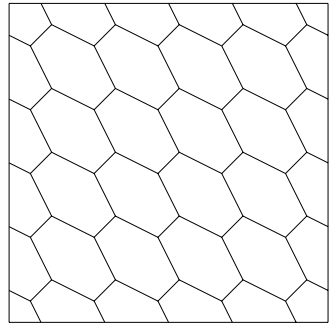

(c)

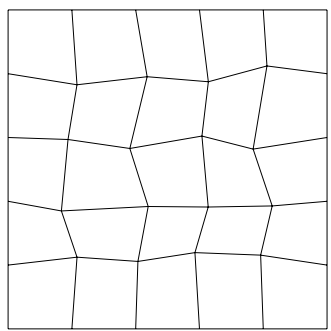

(g)

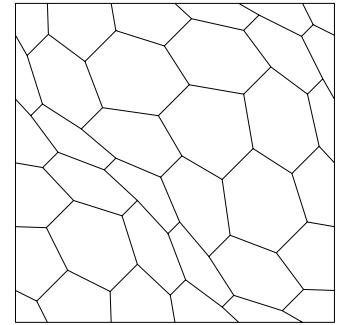

(d)

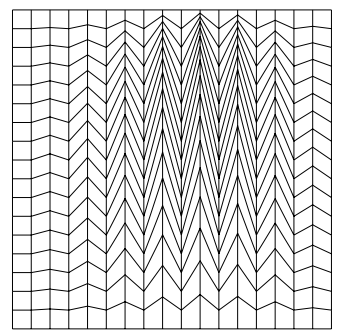

(h)

Fig. 5. First mesh of the sequence used for the patch test; first row: (a) smoothly deformed quadrilaterals; (b) smoothly deformed hexagons; (c) tilted hexagons; (d) smoothly deformed Voronoi; second row: (e) regular octagons and quadrilaterals; (f) regular hexagons; (g) randomly deformed quadrilaterals; (h) highly skewed quadrilaterals.

(i) VEM and the PFEM-VEM always pass the patch test, with relative errors in the $L^{2}$ norm and the $H^{1}$ seminorm of the order of machine precision. In contrast, the PFEM does not pass the patch test.

(ii) VEM and the PFEM-VEM always satisfy the consistency condition $\mathbf{K}_{e} \mathbf{N}=\mathbf{R}$ to machineprecision accuracy, whereas PFEM only does so approximately (see columns $\|\mathbf{E}\|_{\mathcal{F}}$ in Tables 2 and 4).

(iii) VEM, PFEM and PFEM-VEM satisfy the consistency condition $\mathbf{N}^{T} \mathbf{K}_{e} \mathbf{N}=\mathbf{N}^{T} \mathbf{R}$ on all meshes. This result is in agreement with Proposition 3.2.

We interpret the behavior noted in (ii) and (iii) as follows. Proposition 3.2 implies that the projection of the condition $\mathbf{K}_{e} \mathbf{N}=\mathbf{R}$ on the subspace of linear polynomials is always met. Indeed, if we take linear polynomials in the set $\{x y\}$, their degrees of freedom are the columns of matrix $\mathbf{N}$. Instead, the relation $\mathbf{K}_{e} \mathbf{N}=\mathbf{R}$ is guaranteed to be satisfied to machine precision only by the VEM since the VEM is designed to satisfy this relation, whereas for the PFEM it depends on the accuracy of the quadrature rule that is used.

\subsubsection{Convergence for Poisson problem}

Referring to the Poisson boundary-value problem in (3.1), we choose $f(\mathbf{x})$ in accordance with the exact solution $u(\mathbf{x})=16 x y(1-x)(1-y)$. The Dirichlet boundary condition $u=0$ is imposed on 
February 25, 2014 15:35 WSPC/INSTRUCTION FILE review

Polygonal FEM and VEM 19

\begin{tabular}{|c|cc|cc|cc|}
\hline & \multicolumn{2}{|c|}{ VEM } & \multicolumn{2}{c|}{ PFEM } & \multicolumn{2}{c|}{ PFEM-VEM } \\
\cline { 2 - 7 } Mesh & $\frac{\left\|u-u^{h}\right\|_{0, \Omega}}{\|u\|_{0, \Omega}}$ & $\frac{\left|u-u^{h}\right|_{1, \Omega}}{|u|_{1, \Omega}}$ & $\frac{\| u-\left.u^{h}\right|_{0, \Omega}}{\|u\|_{0, \Omega}}$ & $\frac{\left|u-u^{h}\right|_{1, \Omega}}{|u|_{1, \Omega}}$ & $\frac{\| u-\left.u^{h}\right|_{0, \Omega}}{\|u\|_{0, \Omega}}$ & $\frac{\left|u-u^{h}\right|_{1, \Omega}}{|u|_{1, \Omega}}$ \\
\hline $5(\mathrm{a})$ & $3.1 \times 10^{-16}$ & $3.1 \times 10^{-15}$ & $5.6 \times 10^{-4}$ & $4.4 \times 10^{-3}$ & $1.8 \times 10^{-16}$ & $3.1 \times 10^{-15}$ \\
$5(\mathrm{~b})$ & $1.3 \times 10^{-15}$ & $9.6 \times 10^{-15}$ & $3.1 \times 10^{-5}$ & $1.2 \times 10^{-3}$ & $3.3 \times 10^{-16}$ & $9.2 \times 10^{-15}$ \\
$5(\mathrm{c})$ & $3.1 \times 10^{-16}$ & $4.3 \times 10^{-15}$ & $8.2 \times 10^{-5}$ & $1.4 \times 10^{-3}$ & $2.2 \times 10^{-16}$ & $4.5 \times 10^{-15}$ \\
$5(\mathrm{~d})$ & $6.5 \times 10^{-16}$ & $5.0 \times 10^{-15}$ & $8.1 \times 10^{-4}$ & $1.1 \times 10^{-2}$ & $2.2 \times 10^{-16}$ & $4.6 \times 10^{-15}$ \\
$5(\mathrm{e})$ & $5.5 \times 10^{-16}$ & $5.3 \times 10^{-15}$ & $2.9 \times 10^{-4}$ & $6.0 \times 10^{-3}$ & $2.7 \times 10^{-16}$ & $5.1 \times 10^{-15}$ \\
$5(\mathrm{f})$ & $6.0 \times 10^{-16}$ & $3.7 \times 10^{-15}$ & $1.1 \times 10^{-4}$ & $1.8 \times 10^{-3}$ & $2.2 \times 10^{-16}$ & $3.5 \times 10^{-15}$ \\
$5(\mathrm{~g})$ & $1.9 \times 10^{-16}$ & $2.7 \times 10^{-15}$ & $1.0 \times 10^{-5}$ & $9.9 \times 10^{-5}$ & $1.6 \times 10^{-16}$ & $2.6 \times 10^{-15}$ \\
$5(\mathrm{~h})$ & $1.9 \times 10^{-14}$ & $8.3 \times 10^{-14}$ & $5.6 \times 10^{-5}$ & $4.9 \times 10^{-4}$ & $4.1 \times 10^{-15}$ & $2.2 \times 10^{-14}$ \\
\hline
\end{tabular}

Table 1. Relative errors in the $L^{2}$ norm and the $H^{1}$ seminorm for the two-dimensional patch test. Both VEM and PFEM-VEM pass the patch test.

\begin{tabular}{|c|cc|cc|cc|}
\hline \multirow{2}{*}{ Mesh } & \multicolumn{2}{|c|}{ VEM } & \multicolumn{2}{c|}{ PFEM } & \multicolumn{2}{c|}{ PFEM-VEM } \\
\cline { 2 - 7 } & $\|\mathbf{E}\|_{\mathcal{F}}$ & $\left\|\mathbf{N}^{T} \mathbf{E}\right\|_{\mathcal{F}}$ & $\|\mathbf{E}\|_{\mathcal{F}}$ & $\left\|\mathbf{N}^{T} \mathbf{E}\right\|_{\mathcal{F}}$ & $\|\mathbf{E}\|_{\mathcal{F}}$ & $\left\|\mathbf{N}^{T} \mathbf{E}\right\|_{\mathcal{F}}$ \\
\hline $5(\mathrm{a})$ & $9.2 \times 10^{-17}$ & $2.9 \times 10^{-17}$ & $2.8 \times 10^{-3}$ & $1.9 \times 10^{-17}$ & $2.5 \times 10^{-17}$ & $1.6 \times 10^{-17}$ \\
$5(\mathrm{~b})$ & $2.8 \times 10^{-17}$ & $5.1 \times 10^{-18}$ & $1.9 \times 10^{-4}$ & $4.7 \times 10^{-18}$ & $1.1 \times 10^{-17}$ & $2.0 \times 10^{-18}$ \\
$5(\mathrm{c})$ & $4.4 \times 10^{-17}$ & $2.0 \times 10^{-17}$ & $3.0 \times 10^{-4}$ & $1.5 \times 10^{-17}$ & $3.7 \times 10^{-17}$ & $1.4 \times 10^{-17}$ \\
$5(\mathrm{~d})$ & $1.1 \times 10^{-16}$ & $3.7 \times 10^{-17}$ & $5.1 \times 10^{-3}$ & $1.5 \times 10^{-17}$ & $1.9 \times 10^{-17}$ & $1.3 \times 10^{-17}$ \\
$5(\mathrm{e})$ & $4.3 \times 10^{-17}$ & $1.4 \times 10^{-17}$ & $1.2 \times 10^{-3}$ & $1.9 \times 10^{-17}$ & $1.5 \times 10^{-17}$ & $1.0 \times 10^{-17}$ \\
$5(\mathrm{f})$ & $4.1 \times 10^{-17}$ & $1.6 \times 10^{-17}$ & $4.4 \times 10^{-4}$ & $2.3 \times 10^{-17}$ & $2.5 \times 10^{-17}$ & $9.8 \times 10^{-18}$ \\
$5(\mathrm{~g})$ & $5.2 \times 10^{-17}$ & $1.9 \times 10^{-17}$ & $2.6 \times 10^{-5}$ & $1.5 \times 10^{-17}$ & $2.3 \times 10^{-17}$ & $9.1 \times 10^{-18}$ \\
$5(\mathrm{~h})$ & $1.2 \times 10^{-14}$ & $2.5 \times 10^{-15}$ & $3.7 \times 10^{-4}$ & $5.2 \times 10^{-17}$ & $2.1 \times 10^{-16}$ & $6.4 \times 10^{-17}$ \\
\hline
\end{tabular}

Table 2. Approximation errors $\|\mathbf{E}\|_{\mathcal{F}}$ and $\left\|\mathbf{N}^{T} \mathbf{E}\right\|_{\mathcal{F}}$ (Frobenius norm) for the patch test, where $\mathbf{E}=\mathbf{K}_{e} \mathbf{N}-\mathbf{R}$. All the methods manifest linear consistency in agreement with Proposition 3.2.

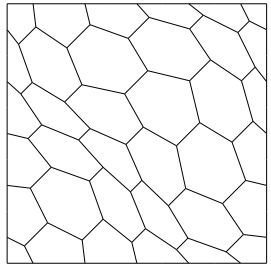

(a)

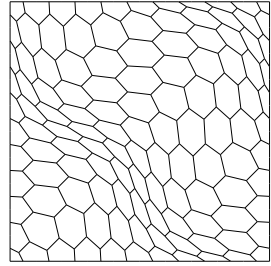

(b)

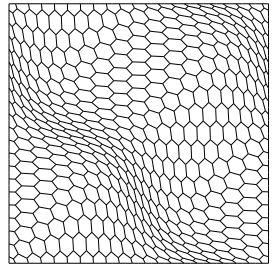

(c)

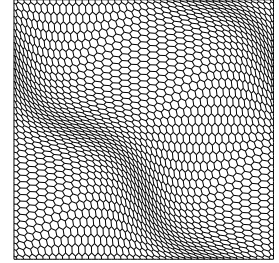

(d)

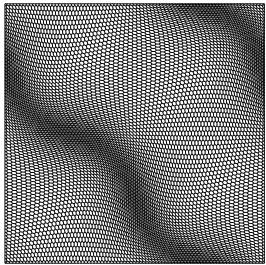

(e)

Fig. 6. Sequence of meshes generated by refining the mesh in Fig. 5d.

$\partial \Omega$. For the VEM, the right-hand side of (3.2) is computed via the approximation

$$
\ell(w)=\int_{\Omega_{e}} f w d \mathbf{x} \approx \int_{\Omega_{e}} \Pi(f) \Pi(w) d \mathbf{x} .
$$


February 25, 2014 15:35 WSPC/INSTRUCTION FILE review

\begin{tabular}{|c|cc|cc|cc|}
\hline \multirow{2}{*}{ Mesh } & \multicolumn{2}{|c|}{ VEM } & \multicolumn{2}{c|}{ PFEM } & \multicolumn{2}{c|}{ PFEM-VEM } \\
\cline { 2 - 6 } & $\frac{\| u-\left.u^{h}\right|_{0, \Omega}}{\|u\|_{0, \Omega}}$ & $\frac{\left|u-u^{h}\right|_{1, \Omega}}{|u|_{1, \Omega}}$ & $\frac{\| u-\left.u^{h}\right|_{0, \Omega}}{\|\left. u\right|_{0, \Omega}}$ & $\frac{\left|u-u^{h}\right|_{1, \Omega}}{|u|_{1, \Omega}}$ & $\frac{\| u-\left.u^{h}\right|_{0, \Omega}}{\|\left. u\right|_{0, \Omega}}$ & $\frac{\left|u-u^{h}\right|_{1, \Omega}}{|u|_{1, \Omega}}$ \\
\hline $6(\mathrm{a})$ & $6.5 \times 10^{-16}$ & $4.8 \times 10^{-15}$ & $6.6 \times 10^{-4}$ & $9.0 \times 10^{-3}$ & $2.0 \times 10^{-16}$ & $4.7 \times 10^{-15}$ \\
$6(\mathrm{~b})$ & $1.8 \times 10^{-15}$ & $9.2 \times 10^{-15}$ & $2.9 \times 10^{-4}$ & $6.3 \times 10^{-3}$ & $2.8 \times 10^{-16}$ & $8.0 \times 10^{-15}$ \\
$6(\mathrm{c})$ & $5.8 \times 10^{-15}$ & $2.3 \times 10^{-14}$ & $6.0 \times 10^{-5}$ & $1.5 \times 10^{-3}$ & $1.3 \times 10^{-15}$ & $1.8 \times 10^{-14}$ \\
$6(\mathrm{~d})$ & $3.7 \times 10^{-14}$ & $1.0 \times 10^{-13}$ & $1.5 \times 10^{-5}$ & $6.3 \times 10^{-4}$ & $1.1 \times 10^{-15}$ & $3.4 \times 10^{-14}$ \\
$6(\mathrm{e})$ & $1.5 \times 10^{-13}$ & $3.8 \times 10^{-13}$ & $3.8 \times 10^{-6}$ & $3.7 \times 10^{-4}$ & $5.3 \times 10^{-15}$ & $6.8 \times 10^{-14}$ \\
$6(\mathrm{f})$ & $6.4 \times 10^{-13}$ & $1.5 \times 10^{-12}$ & $1.0 \times 10^{-6}$ & $2.5 \times 10^{-4}$ & $5.3 \times 10^{-15}$ & $1.4 \times 10^{-13}$ \\
$6(\mathrm{~g})$ & $2.6 \times 10^{-12}$ & $6.3 \times 10^{-12}$ & $2.9 \times 10^{-7}$ & $1.8 \times 10^{-4}$ & $5.0 \times 10^{-14}$ & $3.1 \times 10^{-13}$ \\
$6(\mathrm{~h})$ & $1.3 \times 10^{-11}$ & $2.7 \times 10^{-11}$ & $8.8 \times 10^{-8}$ & $1.3 \times 10^{-4}$ & $6.2 \times 10^{-13}$ & $1.7 \times 10^{-12}$ \\
$6(\mathrm{i})$ & $4.9 \times 10^{-11}$ & $1.2 \times 10^{-10}$ & $2.8 \times 10^{-8}$ & $9.0 \times 10^{-5}$ & $4.5 \times 10^{-12}$ & $1.3 \times 10^{-11}$ \\
\hline
\end{tabular}

Table 3. Relative errors in the $L^{2}$ norm and the $H^{1}$ seminorm for the patch test on a sequence of meshes generated by refining the mesh in Fig. 5d. The first five meshes labeled from 6a to 6e are shown in Fig. 6. Both VEM and PFEM-VEM pass the patch test.

\begin{tabular}{|c|cc|cc|cc|}
\hline \multirow{2}{*}{ Mesh } & \multicolumn{2}{|c|}{ VEM } & \multicolumn{2}{c|}{ PFEM } & \multicolumn{2}{c|}{ PFEM-VEM } \\
\cline { 2 - 7 } & $\|\mathbf{E}\|_{\mathcal{F}}$ & $\left\|\mathbf{N}^{T} \mathbf{E}\right\|_{\mathcal{F}}$ & $\|\mathbf{E}\|_{\mathcal{F}}$ & $\left\|\mathbf{N}^{T} \mathbf{E}\right\|_{\mathcal{F}}$ & $\|\mathbf{E}\|_{\mathcal{F}}$ & $\left\|\mathbf{N}^{T} \mathbf{E}\right\|_{\mathcal{F}}$ \\
\hline $6(\mathrm{a})$ & $1.1 \times 10^{-16}$ & $3.8 \times 10^{-17}$ & $4.2 \times 10^{-3}$ & $1.9 \times 10^{-17}$ & $2.2 \times 10^{-17}$ & $1.3 \times 10^{-17}$ \\
$6(\mathrm{~b})$ & $7.0 \times 10^{-17}$ & $1.3 \times 10^{-17}$ & $1.6 \times 10^{-3}$ & $5.5 \times 10^{-18}$ & $1.2 \times 10^{-17}$ & $4.4 \times 10^{-18}$ \\
$6(\mathrm{c})$ & $4.9 \times 10^{-17}$ & $5.3 \times 10^{-18}$ & $1.4 \times 10^{-4}$ & $1.8 \times 10^{-18}$ & $8.4 \times 10^{-18}$ & $1.4 \times 10^{-18}$ \\
$6(\mathrm{~d})$ & $2.8 \times 10^{-17}$ & $1.6 \times 10^{-18}$ & $4.9 \times 10^{-5}$ & $5.0 \times 10^{-19}$ & $4.4 \times 10^{-17}$ & $3.4 \times 10^{-19}$ \\
$6(\mathrm{e})$ & $1.5 \times 10^{-17}$ & $4.5 \times 10^{-19}$ & $2.2 \times 10^{-5}$ & $1.3 \times 10^{-19}$ & $2.2 \times 10^{-18}$ & $9.6 \times 10^{-20}$ \\
$6(\mathrm{f})$ & $8.7 \times 10^{-18}$ & $1.1 \times 10^{-19}$ & $1.1 \times 10^{-5}$ & $3.3 \times 10^{-20}$ & $1.2 \times 10^{-18}$ & $2.6 \times 10^{-20}$ \\
$6(\mathrm{~g})$ & $5.2 \times 10^{-18}$ & $3.1 \times 10^{-20}$ & $5.7 \times 10^{-6}$ & $8.2 \times 10^{-21}$ & $6.5 \times 10^{-19}$ & $6.8 \times 10^{-21}$ \\
$6(\mathrm{~h})$ & $2.4 \times 10^{-18}$ & $9.5 \times 10^{-21}$ & $2.2 \times 10^{-6}$ & $2.3 \times 10^{-21}$ & $3.1 \times 10^{-19}$ & $1.6 \times 10^{-21}$ \\
$6(\mathrm{i})$ & $1.4 \times 10^{-18}$ & $2.5 \times 10^{-21}$ & $1.4 \times 10^{-6}$ & $6.4 \times 10^{-21}$ & $1.6 \times 10^{-19}$ & $4.5 \times 10^{-22}$ \\
\hline
\end{tabular}

Table 4. Approximation errors $\|\mathbf{E}\|_{\mathcal{F}}$ and $\left\|\mathbf{N}^{T} \mathbf{E}\right\|_{\mathcal{F}}$ (Frobenius norm) for the patch test, where $\mathbf{E}=\mathbf{K}_{e} \mathbf{N}-\mathbf{R}$. Results are computed on the meshes generated by refining the mesh in Fig. 5d. The first five meshes labeled from $6 \mathrm{a}$ to $6 \mathrm{e}$ are shown in Fig. 6. All the schemes manifest linear consistency in agreement with Proposition 3.2.

Using decomposition (3.16), and noting that the second term in this decomposition is orthogonal to constants, we obtain

$$
\begin{aligned}
\int_{\Omega_{e}}\left(\Pi_{0}(f)\right. & \left.+\left(I-\Pi_{0}\right) \tilde{\Pi}(f)\right)\left(\Pi_{0}(w)+\left(I-\Pi_{0}\right) \tilde{\Pi}(w)\right) d \mathbf{x} \\
= & \int_{\Omega_{e}} \Pi_{0}(f) \Pi_{0}(w) d \mathbf{x}+\int_{\Omega_{e}}\left(I-\Pi_{0}\right) \tilde{\Pi}(f)\left(I-\Pi_{0}\right) \tilde{\Pi}(w) d \mathbf{x} .
\end{aligned}
$$


February 25, 2014 15:35 WSPC/INSTRUCTION FILE review

Polygonal FEM and VEM 21

\begin{tabular}{|c|ccc|}
\hline Mesh & $6(a)$ & $6(b)$ & $6(c)$ \\
\hline Nodes & 74 & 244 & 884 \\
$h$ & $3.278 \times 10^{-1}$ & $1.846 \times 10^{-1}$ & $9.686 \times 10^{-2}$ \\
\hline \hline Mesh & $6(d)$ & $6(e)$ & $6(f)$ \\
\hline Nodes & 3364 & 13124 & 51844 \\
$h$ & $4.889 \times 10^{-2}$ & $2.451 \times 10^{-2}$ & $1.226 \times 10^{-2}$ \\
\hline \hline Mesh & $6(g)$ & $6(h)$ & $6(i)$ \\
\hline Nodes & 206084 & 821764 & 3281924 \\
$h$ & $6.131 \times 10^{-3}$ & $3.066 \times 10^{-3}$ & $1.533 \times 10^{-3}$ \\
\hline
\end{tabular}

Table 5. Number of nodes and mesh size $h$ for the meshes generated by refining the mesh in Fig. $5 \mathrm{~d}$ and used in the two-dimensional convergence study. The first five meshes labeled from 6 a to 6 e are shown in Fig. 6.

The argument of the first integral on the right-hand side is a constant function, and using the definition of $\Pi_{0}$ and $\tilde{\Pi}$ yields:

$$
\int_{\Omega_{e}}\left(I-\Pi_{0}\right) \tilde{\Pi}(f)\left(I-\Pi_{0}\right) \tilde{\Pi}(w) d \mathbf{x}=\overline{\nabla f}^{T}\left(\int_{\Omega_{e}}\left(\mathbf{x}-\mathbf{x}_{\Omega_{e}}\right)\left(\mathbf{x}-\mathbf{x}_{\Omega_{e}}\right)^{T} d \mathbf{x}\right) \overline{\nabla w}=\mathcal{O}\left(h^{2}\right),
$$

where $\overline{\nabla f}$ and $\overline{\nabla w}$ are some suitable constant approximation of the gradients of $f$ and $w$, and this term is proportional to $h^{2}$. Thus, the VEM approximation for the right-hand side of (3.2) is:

$$
\ell(w)=\Pi_{0}(f) \Pi_{0}(w)\left|\Omega_{e}\right|+\mathcal{O}\left(h^{2}\right) .
$$

In (4.1), we evaluate $\Pi_{0}(w)$ by using $(3.4 c)$, and $\Pi_{0}(f)$ by the cell-average of $f$ on $\Omega_{e}$. For the PFEM-VEM, the right-hand side of (3.2) can be evaluated through (4.1) or by evaluating the integral in (3.2) by a quadrature rule as for the PFEM. The results shown in this section are obtained by adopting this latter approach. The computation of the element force vector in PFEM and PFEM-VEM is carried out using 1-point quadrature rule in each triangle-partition of a polygonal element. Convergence study using the VEM, PFEM and PFEM-VEM is conducted on the meshes shown in Fig. 6. The mesh parameters are presented in Table 5. The numerical results for the relative error norms are listed in Tables 6 and 7. Plots of the rate of convergence of the three methods are depicted in Fig. 7. We observe that all methods deliver the optimal rate of convergence in the $L^{2}$ norm and the $H^{1}$ seminorm.

\subsection{Three-dimensional Poisson problems}

\subsubsection{Patch test}

We consider the Laplace equation in a unit cube with linear Dirichlet boundary conditions. We choose $f(\mathbf{x})=0$ and $g(\mathbf{x})=1-3 x+4 y-5 z$ in (3.1). The exact solution is: $u(\mathbf{x})=g(\mathbf{x})$. We perform numerical computations with PFEM and the combined PFEM-VEM formulation of Section 3.3. Numerical integration in PFEM is directly performed by partitioning each physical polyhedron into subtetrahedra, and using one-point integration rule in each subtetrahedra. For the PFEM-VEM computations, the PFEM stiffness matrix $\mathbf{K}_{e}^{t}(t=1)$ is used in (3.26). For the patch test, the sequence of meshes shown in Fig. 8 are used. Numerical results for the relative error norms are 
February 25, 2014 15:35 WSPC/INSTRUCTION FILE review

\begin{tabular}{|c|cc|cc|cc|}
\hline & \multicolumn{2}{|c|}{ VEM } & \multicolumn{2}{c|}{ PFEM } & \multicolumn{2}{c|}{ PFEM-VEM } \\
\cline { 2 - 7 } Mesh & $\frac{\left\|u-u^{h}\right\|_{0, \Omega}}{\|u\|_{0, \Omega}}$ & Rate & $\frac{\left\|u-u^{h}\right\|_{0, \Omega}}{\|u\|_{0, \Omega}}$ & Rate & $\frac{\left\|u-u^{h}\right\|_{0, \Omega}}{\|u\|_{0, \Omega}}$ & Rate \\
\hline $6(\mathrm{a})$ & $8.71 \times 10^{-2}$ & - & $6.28 \times 10^{-2}$ & - & $5.09 \times 10^{-2}$ & - \\
$6(\mathrm{~b})$ & $2.95 \times 10^{-2}$ & 1.89 & $1.91 \times 10^{-2}$ & 2.07 & $1.48 \times 10^{-2}$ & 2.15 \\
$6(\mathrm{c})$ & $8.52 \times 10^{-3}$ & 1.92 & $5.16 \times 10^{-3}$ & 2.02 & $3.96 \times 10^{-3}$ & 2.05 \\
$6(\mathrm{~d})$ & $2.27 \times 10^{-3}$ & 1.94 & $1.32 \times 10^{-3}$ & 1.98 & $1.01 \times 10^{-3}$ & 1.99 \\
$6(\mathrm{e})$ & $5.82 \times 10^{-4}$ & 1.99 & $3.36 \times 10^{-4}$ & 1.99 & $2.55 \times 10^{-4}$ & 1.99 \\
$6(\mathrm{f})$ & $1.47 \times 10^{-4}$ & 1.98 & $8.43 \times 10^{-5}$ & 1.99 & $6.40 \times 10^{-5}$ & 2.00 \\
$6(\mathrm{~g})$ & $3.70 \times 10^{-5}$ & 1.99 & $2.11 \times 10^{-5}$ & 2.00 & $1.60 \times 10^{-5}$ & 2.00 \\
$6(\mathrm{~h})$ & $9.28 \times 10^{-6}$ & 2.00 & $5.26 \times 10^{-6}$ & 2.00 & $4.01 \times 10^{-6}$ & 2.00 \\
$6(\mathrm{i})$ & $2.32 \times 10^{-6}$ & 2.00 & $1.31 \times 10^{-6}$ & 2.00 & $1.00 \times 10^{-6}$ & 2.00 \\
\hline
\end{tabular}

Table 6. Relative errors in the $L^{2}$ norm for the two-dimensional Poisson problem using VEM, PFEM and PFEMVEM. The meshes are generated by refining the mesh in Fig. $5 \mathrm{~d}$ and used in the two-dimensional convergence study. The first five meshes labeled from 6a to 6e are shown in Fig. 6.

\begin{tabular}{|c|cc|cc|cc|}
\hline \multirow{2}{*}{ Mesh } & \multicolumn{2}{|c|}{ VEM } & \multicolumn{2}{c|}{ PFEM } & \multicolumn{2}{c|}{ PFEM-VEM } \\
\cline { 2 - 7 } & $\frac{\left|u-u^{h}\right|_{1, \Omega}}{|u|_{1, \Omega}}$ & Rate & $\frac{\left|u-u^{h}\right|_{1, \Omega}}{|u|_{1, \Omega}}$ & Rate & $\frac{\left|u-u^{h}\right|_{1, \Omega}}{|u|_{1, \Omega}}$ & Rate \\
\hline $6(\mathrm{a})$ & $2.44 \times 10^{-1}$ & - & $2.40 \times 10^{-1}$ & - & $2.43 \times 10^{-1}$ & - \\
$6(\mathrm{~b})$ & $1.36 \times 10^{-1}$ & 1.02 & $1.34 \times 10^{-1}$ & 1.01 & $1.35 \times 10^{-1}$ & 1.02 \\
$6(\mathrm{c})$ & $7.02 \times 10^{-2}$ & 1.03 & $6.98 \times 10^{-2}$ & 1.01 & $7.01 \times 10^{-2}$ & 1.02 \\
$6(\mathrm{~d})$ & $3.55 \times 10^{-2}$ & 1.00 & $3.54 \times 10^{-2}$ & 0.99 & $3.55 \times 10^{-2}$ & 1.00 \\
$6(\mathrm{e})$ & $1.78 \times 10^{-2}$ & 1.00 & $1.78 \times 10^{-2}$ & 0.99 & $1.78 \times 10^{-2}$ & 0.99 \\
$6(\mathrm{f})$ & $8.95 \times 10^{-3}$ & 1.00 & $8.95 \times 10^{-3}$ & 0.99 & $8.95 \times 10^{-3}$ & 1.00 \\
$6(\mathrm{~g})$ & $4.48 \times 10^{-3}$ & 1.00 & $4.49 \times 10^{-3}$ & 1.00 & $4.48 \times 10^{-3}$ & 1.00 \\
$6(\mathrm{~h})$ & $2.24 \times 10^{-3}$ & 1.00 & $2.25 \times 10^{-3}$ & 0.99 & $2.24 \times 10^{-3}$ & 1.00 \\
$6(\mathrm{i})$ & $1.12 \times 10^{-3}$ & 1.00 & $1.13 \times 10^{-3}$ & 0.99 & $1.12 \times 10^{-3}$ & 1.00 \\
\hline
\end{tabular}

Table 7. Relative errors in the $H^{1}$ seminorm for the two-dimensional Poisson problem using VEM, PFEM and PFEMVEM. The meshes are generated by refining the mesh in Fig. $5 \mathrm{~d}$ and used in the two-dimensional convergence study. The first five meshes labeled from 6a to 6e are shown in Fig. 6.

presented in Table 8. The PFEM does not pass the patch test, whereas the PFEM-VEM passes the patch test.

\subsubsection{Convergence for Poisson problem}

Referring to the Poisson boundary-value problem in (3.1), we choose $f(\mathbf{x})$ in accordance with the exact solution $u(\mathbf{x})=64 x y z(1-x)(1-y)(1-z)$, and the Dirichlet boundary condition $u=0$ is imposed on $\partial \Omega$. The computation of the element force vector in PFEM and PFEM-VEM is carried out using 1-point quadrature rule in each subtetrahedra of a partitioned-polyhedron. Convergence study using PFEM and PFEM-VEM is conducted on the meshes shown in Fig. 8. The numerical 


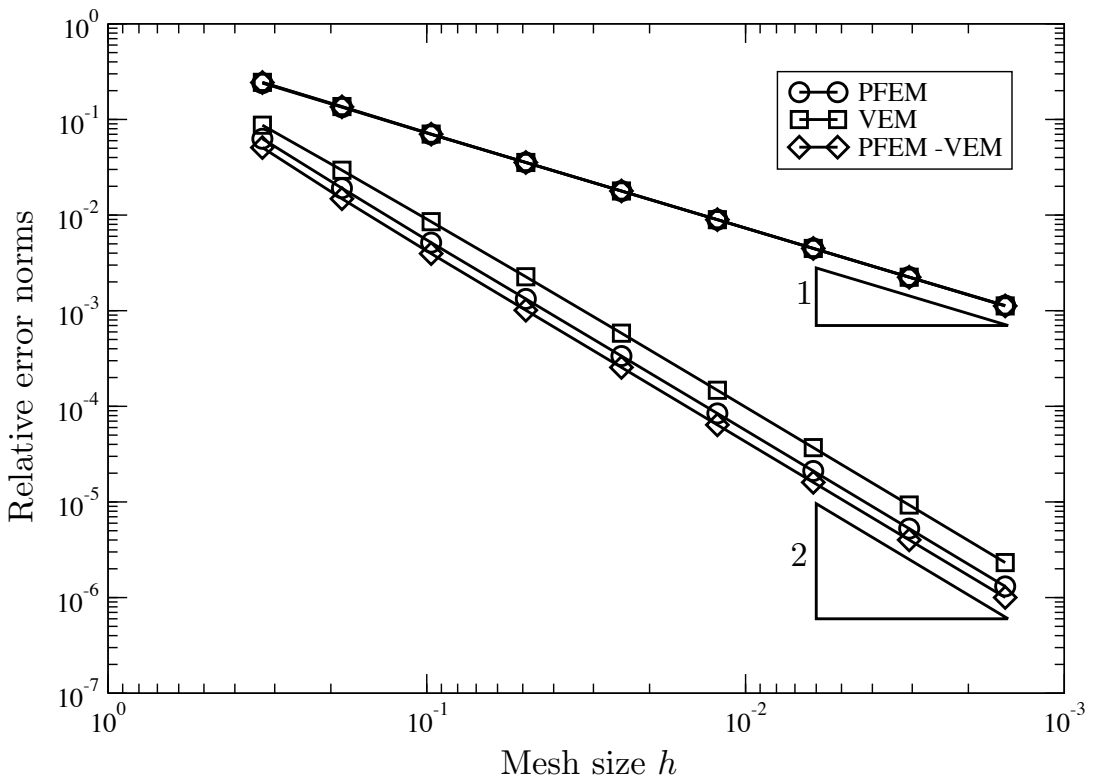

Fig. 7. Rate of convergence for the two-dimensional Poisson problem using VEM, PFEM and PFEM-VEM. All methods deliver optimal convergence rates of 2 and 1 in the $L^{2}$ norm and the $H^{1}$ seminorm, respectively.

\begin{tabular}{|c|c|c|cc|cc|}
\hline & \multirow{2}{*}{ Mesh } & \multirow{2}{*}{ Nodes } & & \multicolumn{2}{|c|}{ PFEM } & \multicolumn{2}{c|}{ PFEM-VEM } \\
\cline { 4 - 7 } & & & $\frac{\left\|u-u^{h}\right\|_{0, \Omega}}{\|u\|_{0, \Omega}}$ & $\frac{\left|u-u^{h}\right|_{1, \Omega}}{|u|_{1, \Omega}}$ & $\frac{\left\|u-u^{h}\right\|_{0, \Omega}}{\|u\|_{0, \Omega}}$ & $\frac{\left|u-u^{h}\right|_{1, \Omega}}{|u|_{1, \Omega}}$ \\
\hline $8(\mathrm{a})$ & 275 & 0.530 & $1.1 \times 10^{-3}$ & $7.7 \times 10^{-3}$ & $1.1 \times 10^{-15}$ & $1.8 \times 10^{-14}$ \\
$8(\mathrm{~b})$ & 1177 & 0.347 & $6.7 \times 10^{-4}$ & $8.9 \times 10^{-3}$ & $2.3 \times 10^{-15}$ & $4.1 \times 10^{-14}$ \\
$8(\mathrm{c})$ & 13489 & 0.175 & $2.8 \times 10^{-4}$ & $8.8 \times 10^{-3}$ & $3.9 \times 10^{-14}$ & $1.7 \times 10^{-12}$ \\
8(d) & 106327 & 0.083 & $1.4 \times 10^{-4}$ & $9.0 \times 10^{-3}$ & $1.8 \times 10^{-13}$ & $1.5 \times 10^{-11}$ \\
\hline
\end{tabular}

Table 8. Relative errors in the $L^{2}$ norm and the $H^{1}$ seminorm for the three-dimensional patch test. PFEM does not pass the patch test, whereas PFEM-VEM passes the patch test.

results for the relative error norms are listed in Table 9, and the plots of the rate of convergence of the methods are depicted in Fig. 9. On the meshes chosen, we observe that both methods deliver the optimal rate of convergence in the $L^{2}$ norm and the $H^{1}$ seminorm. However, unlike the two-dimensional case, note that the error in the $H^{1}$ seminorm for the patch test in three dimensions with PFEM stagnates to $O\left(10^{-3}\right)$ (see Table 8), and therefore it is expected that the rate of convergence with the PFEM will deteriorate on more refined meshes. This inference is also consistent with the analysis for the patch test presented in Reference [67]. 


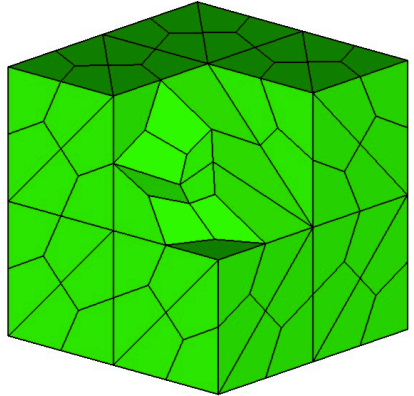

(a)

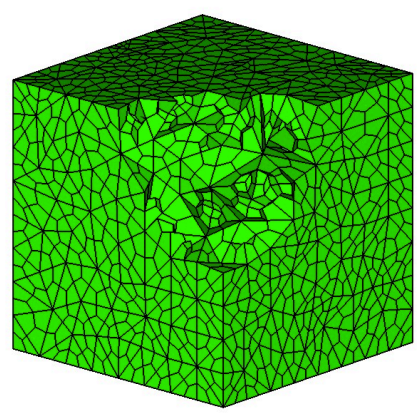

(c)

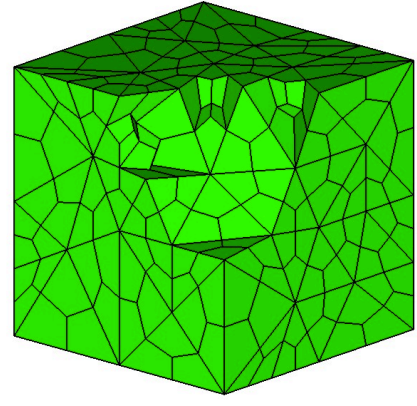

(b)

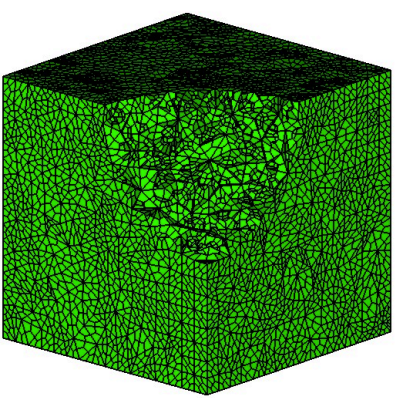

(d)

Fig. 8. Sequence of polyhedral meshes used for the patch test and for the convergence study on the Poisson problem. The cells are hexahedra obtaining by partitioning tetrahedral cells into four subcells. A portion of the mesh around the vertex $(1,1,1)$ has been removed to show the internal structure.

\begin{tabular}{|c|cccc|cccc|}
\hline \multirow{3}{*}{ Mesh } & \multicolumn{4}{|c|}{ PFEM } & \multicolumn{4}{c|}{ PFEM-VEM } \\
\cline { 2 - 6 } & $\frac{\left\|u-u^{h}\right\|_{0, \Omega}}{\|u\|_{0, \Omega}}$ & Rate & $\frac{\left\|u-u^{h}\right\|_{1, \Omega}}{\|u\|_{1, \Omega}}$ & Rate & $\frac{\left\|u-u^{h}\right\|_{0, \Omega}}{\|u\|_{0, \Omega}}$ & Rate & $\frac{\left\|u-u^{h}\right\|_{1, \Omega}}{\|u\|_{1, \Omega}}$ & Rate \\
\hline 8(a) & $1.8 \times 10^{-1}$ & - & $3.6 \times 10^{-1}$ & - & $1.8 \times 10^{-1}$ & - & $3.6 \times 10^{-1}$ & - \\
8(b) & $5.1 \times 10^{-2}$ & 2.95 & $1.8 \times 10^{-1}$ & 1.69 & $5.0 \times 10^{-2}$ & 3.01 & $1.8 \times 10^{-1}$ & 1.68 \\
$8(\mathrm{c})$ & $9.4 \times 10^{-3}$ & 2.49 & $7.5 \times 10^{-2}$ & 1.26 & $9.1 \times 10^{-3}$ & 2.49 & $7.6 \times 10^{-2}$ & 1.25 \\
8(d) & $2.1 \times 10^{-3}$ & 2.01 & $3.6 \times 10^{-2}$ & 0.98 & $2.1 \times 10^{-3}$ & 1.99 & $3.6 \times 10^{-2}$ & 1.01 \\
\hline
\end{tabular}

Table 9. Relative errors in the $L^{2}$ norm and the $H^{1}$ seminorm for the three-dimensional Poisson problem.

\section{Concluding Remarks}

In this paper, we first presented an overview of generalized barycentric coordinates, with emphasis on Wachspress and maximum-entropy coordinates. Recently, mimetic finite-difference schemes on 


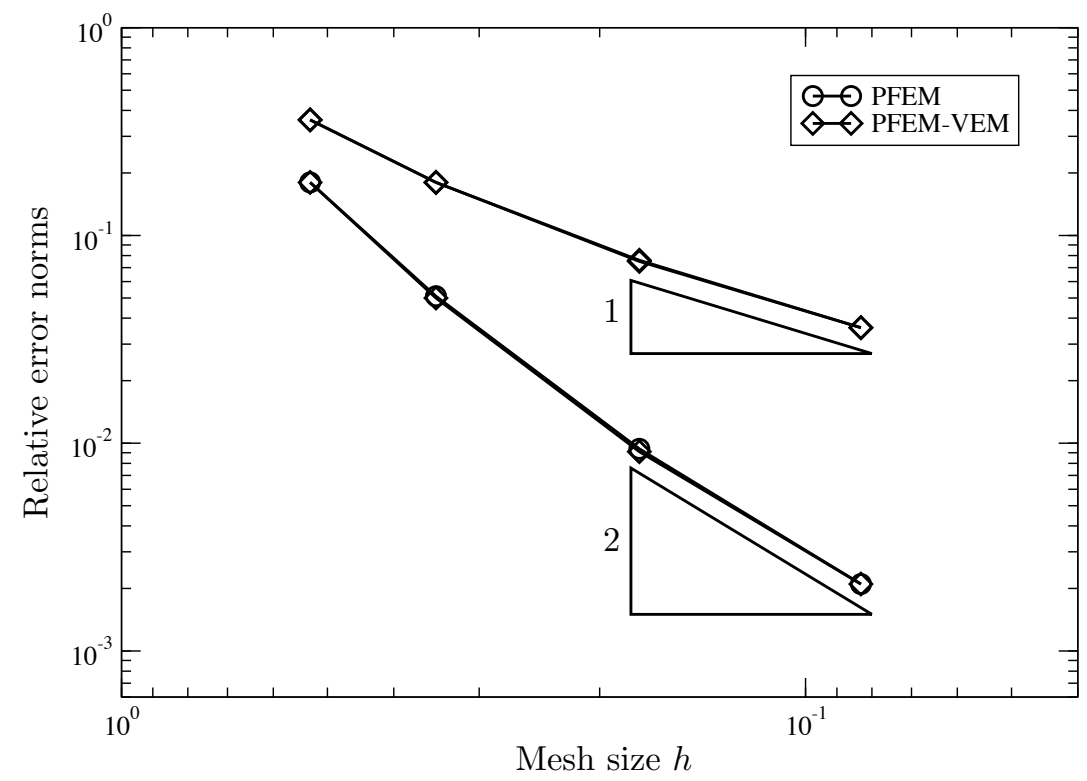

Fig. 9. Rate of convergence for the thres-dimensional Poisson problem using PFEM and PFEM-VEM. All methods deliver optimal convergence rates of 2 and 1 in the $L^{2}$ norm and the $H^{1}$ seminorm, respectively.

arbitrary polygonal meshes were cast in a variational framework - the method so devised was coined as the Virtual Element Method (VEM), since it did not require the explicit construction of basis functions. In the VEM, a projection operator is used to realize the decomposition of the stiffness matrix into two terms: a consistent matrix that is known, and a stability matrix that must be positive semi-definite and which is only required to scale like the consistent matrix. We appealed to the exact decomposition in the VEM to propose a robust and efficient Galerkin method using Wachspress coordinates on polygonal and polyhedral elements. To this end, we used numerical quadrature to compute the stability matrix.

As a model problem, we considered the Poisson equation with Dirichlet boundary conditions. In the numerical computations, we compared the accuracy of the VEM, polygonal/polyhedral finite element method (PFEM), and the combined PFEM-VEM approach. First, the patch test was assessed for the Laplace equation in $\mathbb{R}^{2}$ and $\mathbb{R}^{3}$, with linear Dirichlet boundary conditions. We showed that PFEM does not pass the patch test, whereas both VEM and PFEM-VEM pass the patch test. Then, we established that the PFEM-VEM approach delivered optimal rates of convergence in the $L^{2}$ norm and the $H^{1}$ seminorm for the two- and three-dimensional Poisson equation with homogeneous Dirichlet boundary conditions. On combining the virtual element formulation to barycentric finite elements, a robust and efficient approach has been realized for solving second-order elliptic problems on polygonal and polyhedral meshes. 


\section{Acknowledgment}

The work of GM was partially supported by the National Nuclear Security Administration of the U.S. Department of Energy at Los Alamos National Laboratory under Contract No. DE-AC5206NA25396 and the DOE Office of Science Advanced Scientific Computing Research (ASCR) Program in Applied Mathematics. NS gratefully acknowledges the research support of the National Science Foundation through contract grant CMMI-1334783 to the University of California at Davis. NS also thanks Michael Floater, Andrew Gillette and Kai Hormann for many helpful discussions.

\section{References}

1. A. F. Möbius. Der barycentrische Calcul. Johann Ambrosius Barth, Leipzig, 1827.

2. E. L. Wachspress. A Rational Finite Element Basis, volume 114 of Mathematics in Science and Engineering. Academic Press, 1975.

3. H. S. M. Coxeter. Introduction to Geometry. John Wiley and Sons, New York, N.Y., 1961.

4. M. Meyer, H. Lee, A. Barr, and M. Desbrun. Generalized barycentric coordinates on irregular polygons. Journal of Graphics Tools, 7(1):13-22, 2002.

5. G. Dasgupta. Interpolants within convex polygons: Wachspress' shape functions. Journal of Aerospace Engineering, 16(1):1-8, 2003.

6. G. Dasgupta. Integration within polygonal finite elements. Journal of Aerospace Engineering, 16(1):918, 2003.

7. M. S. Floater. Mean value coordinates. Computer Aided Geometric Design, 20(1):19-27, 2003.

8. M. S. Floater, K. Hormann, and G. Kós. A general construction of barycentric coordinates over convex polygons. Advances in Computational Mathematics, 24(1-4):311-331, 2006.

9. T. Ju, P. Liepa, and J. Warren. A general geometric construction of coordinates in a convex simplicial polytope. Computer Aided Geometric Design, 24(3):161-178, 2007.

10. S. Schaefer, T. Ju, and J. Warren. A unified, integral construction for coordinates over closed curves. Computer Aided Geometric Design, 24(8-9):481-493, 2007.

11. R. Sibson. A vector identity for the Dirichlet tesselation. Mathematical Proceedings of the Cambridge Philosophical Society, 87:151-155, 1980.

12. N. H. Christ, R. Friedberg, and T. D. Lee. Weights of links and plaquettes in a random lattice. Nuclear Physics B, 210(3):337-346, 1982.

13. H. Hiyoshi and K. Sugihara. Two generalizations of an interpolant based on Voronoi diagrams. International Journal of Shape Modeling, 5(2):219-231, 1999.

14. U. Pinkall and K. Polthier. Computing discrete minimal surfaces and their conjugates. Experimental Mathematics, 2(1):15-36, 1993.

15. M. Eck, T. DeRose, T. Duchamp, H. Hoppe, M. Lounsbery, and W. Stuetzle. Multiresolution analysis of arbitrary meshes. In Proceedings of SIGGRAPH 95, pages 173-182. ACM Press, 1995.

16. E. A. Malsch, J. J. Lin, and G. Dasgupta. Smooth two dimensional interpolants: a recipe for all polygons. Journal of Graphics Tools, 10(2):27-39, 2005.

17. N. Sukumar and E. A. Malsch. Recent advances in the construction of polygonal finite element interpolants. Archives of Computational Methods in Engineering, 13(1):129-163, 2006.

18. P. Joshi, M. Meyer, T. DeRose, B. Green, and T. Sanocki. Harmonic coordinates for character articulation. ACM Transactions on Graphics, 26(3):71/1-71/9, 2007. Proceedings of ACM SIGGRAPH 2007.

19. R. M. Rustamov. Boundary element formulation of harmonic coordinates. Technical report, Department of Mathematics, Purdue University, 2008.

20. Y. Lipman, J. Kopf, D. Cohen-Or, and D. Levin. GPU-assisted positive mean value coordinates for mesh deformations. In Geometry Processing, Eurographics Symposium Proceedings, pages 117-123. Eurographics Association, 2007. 
21. K. Hormann and N. Sukumar. Maximum entropy coordinates for arbitrary polytopes. Computer Graphics Forum, 27(5):1513-1520, 2008.

22. O. Weber, M. Ben-Chen, and C. Gotsman. Complex barycentric coordinates with applications to planar shape deformation. Computer Graphics Forum (Proceedings of Eurographics), 28(2), 2009.

23. O. Weber, M. Ben-Chen, C. Gotsman, and K. Hormann. A complex view of barycentric mappings. Computer Graphics Forum, 30(5):1533-1542, 2011.

24. J. Manson and S. Schaefer. Movine least squares coordinates. Computer Graphics Forum, 29(5):15171524, 2010.

25. X.-Y. Li and S.-M. Hu. Poisson coordinates. IEEE Transactions on Visualization and Computer Graphics, 19:344-352, 2013.

26. X.-Y. Li, T. Ju, and S.-M. Hu. Cubic mean value coordinates. ACM Transactions on Graphics, $32(4): 126,2013$.

27. J. Warren. Barycentric coordinates for convex polytopes. Advances in Computational Mathematics, 6(2):97-108, 1996.

28. E. L. Wachspress. Rational bases for convex polyhedra. Computers $\&$ Mathematics with Applications, 59(6):1953-1956, 2010.

29. E. L. Wachspress. Barycentric coordinates for polytopes. Computers 6 Mathematics with Applications, 61(11):3319-3321, 2011.

30. M. S. Floater, G. Kós, and M. Reimers. Mean value coordinates in 3D. Computer Aided Geometric Design, 22(7):623-631, 2005.

31. J. Warren. On the uniqueness of barycentric coordinates. In Contemporary Mathematics, Proceedings of AGGM02, pages 93-99, 2003.

32. A. Belyaev. On transfinite barycentric coordinates. In Geometry Processing, Eurographics Symposium Proceedings, pages 89-99. Eurographics Association, 2006.

33. C. Dyken and M. S. Floater. Transfinite mean value interpolation. Computer Aided Geometric Design, 26(1):117-134, 2009.

34. T. Langer, A. Belyaev, and H.-P. Seidel. Spherical barycentric coordinates. In Geometry Processing, Eurographics Symposium Proceedings, pages 81-88. Eurographics Association, 2006.

35. T. Langer and H.-P. Seidel. Higher order barycentric coordinates. Computer Graphics Forum, 27(2):459-466, 2008. Proceedings of Eurographics 2008.

36. M. Wardetzky, S. Mathur, F. Kälberer, and E. Grinspun. Discrete Laplace operators: No free lunch. In Symposium on Geometry Processing, pages 33-37, Jul 2007.

37. M. Alexa and M. Wardetzky. Discrete Laplacian on general polygonal meshes. ACM Transactions on Graphics, 30(4), 2011. Proceedings of ACM SIGGRAPH 2011.

38. R. Rustamov, Y. Lipman, and T. Funkhouser. Interior distance using barycentric coordinates. Computer Graphics Forum, 28(5):1279-1288, 2009.

39. N. Sukumar and A. Tabarraei. Conforming polygonal finite elements. Int. J. Numer. Methods Engrg., 61(12):2045-2066, 2004.

40. N. Sukumar. Construction of polygonal interpolants: a maximum entropy approach. Int. J. Numer. Methods Engrg., 61(12):2159-2181, 2004.

41. A. Tabarraei and N. Sukumar. Application of polygonal finite elements in linear elasticity. Int. J. Comp. Meth., 3(4):503-520, 2006.

42. P. Milbradt and T. Pick. Polytope finite elements. Int. J. Numer. Methods Engrg., 73(12):1811-1835, 2007.

43. M. Wicke, M. Botsch, and M. Gross. A finite element method on convex polyhedra. Computer Graphics Forum, 26(3):355-364, 2007. Proceedings of Eurographics 2007.

44. S. Martin, P. Kaufmann, M. Botsch, M. Wicke, and M. Gross. Polyhedral finite elements using harmonic basis functions. Computer Graphics Forum, 27(5):1521-1529, 2008.

45. S. Weißer. Residual error estimates for BEM-based FEM on polygonal meshes. Numerische Mathematik, 118:765-788, 2011. 
46. S. Rjasanow and S. Weißer. Higher order BEM-based FEM on polygonal meshes. SIAM J. Numer. Anal., 50(5):2357-2378, 2012.

47. M. Kraus and P. Steinmann. Finite element formulations for 3D convex polyhedra in nonlinear continuum mechanics. Computer Assisted Methods in Engineering and Science, 19:121-134, 2012.

48. M. Kraus, A. Rajagopal, and P. Steinmann. Investigations on the polygonal finite element method: Constrained adaptive Delaunay tessellation and conforming interpolants. Computers 85 Structures, 120:33-46, 2013.

49. J. E. Bishop. A displacement-based finite-element formulation for general polyhedra using harmonic shape functions. Int. J. Numer. Methods Engrg., 2013. in press.

50. C. Talischi, A. Pereira, G. H. Paulino, I. F. M. Menezes, and M. S. Carvalho. Polygonal finite elements for incompressible fluid flow. Int. J. Numer. Methods Fluids, 2013. in press. DOI: 10.1002/fld.3843.

51. A. Tabarraei and N. Sukumar. Extended finite element method on polygonal and quadtree meshes. Comp. Meth. Appl. Mech. Engrg., 197(5):425-438, 2008.

52. J. E. Bishop. Simulating the pervasive fracture of materials and structures using randomly closed packed Voronoi tessellations. Comp. Mech., 44(4):455-471, 2009.

53. E. T. Ooi, C. M. Song, F. Tin-Loi, and Z. J. Yang. Polygon scaled boundary finite elements for crack propagation modeling. Int. J. Numer. Methods Engrg., 91(3):319-342, 2012.

54. C. Talischi, G. H. Paulino, and C. H. Le. Honeycomb Wachspress finite elements for structural topology optimization. Struct. Multidisc. Optim., 37(6):569-583, 2009.

55. C. Talischi, G. H. Paulino, A. Pereira, and I. F. M. Menezes. Polygonal finite elements for topology optimization: A unifying paradigm. Int. J. Numer. Methods Engrg., 82(6):671-698, 2010.

56. C. Talischi, G. H. Paulino, A. Pereira, and I. F. M. Menezes. PolyTop: A Matlab implementation of a general topology framework using unstructured polygonal finite element meshes. Struct. Multidisc. Optim., 45(3):329-357, 2012.

57. D. Sieger, P. Alliez, and M. Botsch. Optimizing Voronoi diagrams for polygonal finite elements. Proceedings of the 19th International Meshing Roundtable, pages 335-350, 2010.

58. C. Talischi, G. H. Paulino, A. Pereira, and I. F. M. Menezes. PolyMesher: A general-purpose mesh generator for polygonal elements written in Matlab. Struct. Multidisc. Optim., 45(3):309-328, 2012.

59. M. S. Ebeida and S. A. Mitchell. Uniform random Voronoi meshes. In Proceedings of the 20th International Meshing Roundtable, pages 258-275, Paris, France, 2011.

60. M. S. Ebeida, A. A. Davidson, A. Patney, P. M. Knupp, S. A. Mitchell, and J. D. Owens. Efficient maximal Poisson-disk sampling. In ACM Transactions on Graphics (TOG), volume 30, page 49, 2011.

61. M. S. Ebeida, S. A. Mitchell, A. Patney, A. A. Davidson, and J. D. Owens. A simple algorithm for maximal Poisson-disk sampling in high dimensions. Computer Graphics Forum, 31(2):785-794, 2012.

62. A. Rand, A. Gillete, and C. Bajaj. Interpolation error estimates for mean value coordinates over convex polygons. Advances in Computational Mathematics, 2013. DOI: 10.1007/s10444-012-9282-z.

63. A. Rand, A. Gillete, and C. Bajaj. Error estimates for generalized barycentric interpolation. Advances in Computational Mathematics, 37(3):417-439, 2012.

64. L. Beirão da Veiga, F. Brezzi, A. Cangiani, G. Manzini, L. D. Marini, and A. Russo. Basic principles of virtual element methods. Math. Models Methods Appl. Sci., 23:199, 2013.

65. F. Brezzi and L. D. Marini. Virtual element method for plate bending problems. Comp. Meth. Appl. Mech. Engrg., 253:455-462, 2013.

66. L. Beirão da Veiga, F. Brezzi, and L. D. Marini. Virtual elements for linear elasticity problems. SIAM J. Numer. Anal., 51(2):794-812, 2013.

67. C. Talischi and G. H. Paulino. Addressing integration error for polygonal finite elements through polynomial projections: A patch test connection. Math. Models Methods Appl. Sci., 2013. in press.

68. J. F. Epperson. On the Runge example. The American Mathematical Monthly, 94(4):329-341, 1987.

69. G. Dasgupta and E. L. Wachspress. The adjoint for an algebraic finite element. Computers $\&$ Mathematics with Applications, 55(9):1988-1997, 2008.

70. M. S. Floater, A. Gillette, and N. Sukumar. Gradient bounds for Wachspress coordinates on poly- 
February 25, 2014 15:35 WSPC/INSTRUCTION FILE review

Polygonal FEM and VEM 2

topes. 2013. Preprint available at http://arxiv.org/abs/1306.4385.

71. K. Hormann and M. S. Floater. Mean value coordinates for arbitrary planar polygons. ACM Transactions on Graphics, 25(4):1424-1441, 2006.

72. C. E. Shannon. A mathematical theory of communication. The Bell Systems Technical Journal, 27:379-423, 1948 .

73. S. Kullback and R. A. Leibler. On information and sufficiency. Annals of Mathematical Statistics, 22(1):79-86, 1951.

74. N. Sukumar and R. W. Wright. Overview and construction of meshfree basis functions: from moving least squares to entropy approximants. Int. J. Numer. Methods Engrg., 70(2):181-205, 2006.

75. N. Sukumar. Quadratic serendipity maximum-entropy shape functions for arbitrary planar polygons. Comp. Meth. Appl. Mech. Engrg., 263:27-41, 2013.

76. M. Arroyo and M. Ortiz. Local maximum-entropy approximation schemes: a seamless bridge between finite elements and meshfree methods. Int. J. Numer. Methods Engrg., 65(13):2167-2202, 2006.

77. F. Greco and N. Sukumar. Derivatives of maximum-entropy basis functions on the boundary: Theory and computations. Int. J. Numer. Methods Engrg., 94(12):1123-1149, 2013.

78. N. Sukumar, B. Moran, and T. Belytschko. The natural element method in solid mechanics. Int. J. Numer. Methods Engrg., 43(5):839-887, 1998.

79. T. Ju, S. Schaefer, J. Warren, and M. Desbrun. A geometric construction of coordinates for convex polyhedra using polar duals. In M. Desbrun and H. Pottman, editors, Geometry Processing 2005, pages 181-186. Eurographics Association, 2005.

80. T. Ju, S. Schaefer, and J. Warren. Mean value coordinates for closed triangular meshes. ACM Transactions on Graphics, 24(3):561-566, 2005. Proceedings of ACM SIGGRAPH 2005.

81. T. Langer, A. Belyaev, and H.-P. Seidel. Mean value coordinates for arbitrary spherical polygons and polyhedra in $\mathbb{R}^{3}$. In Patrick Chenin, Tom Lyche, and Larry L. Schumaker, editors, Curve and Surface Design: Avignon 2006, Modern Methods in Applied Mathematics, pages 193-202. Nashboro Press, Brentwood, TN, 2007.

82. S. Ghosh and S. N. Mukhopadhyay. A material based finite-element analysis of heterogeneous media involving Dirichlet tessellations. Comp. Meth. Appl. Mech. Engrg., 104(2):211-247, 1993.

83. S. Ghosh and S. Moorthy. Elastic-plastic analysis of arbitrary heterogeneous materials with the Voronoi cell finite-element method. Comp. Meth. Appl. Mech. Engrg., 121(1-4):373-409, 1995.

84. S. Ghosh. Micromechanical Analysis and Multi-Scale Modeling Using the Voronoi Cell Finite Element Method. CRC Press/Taylor \& Francis Group, Boca Raton, FL, 2011.

85. M. M. Rashid and P. M. Gullett. On a finite element method with variable element topology. Comp. Meth. Appl. Mech. Engrg., 190(11-12):1509-1527, 2000.

86. M. M. Rashid and M. Selimotic. A three-dimensional finite element method with arbitrary polyhedral elements. Int. J. Numer. Methods Engrg., 67:226-252, 2006.

87. M. M. Rashid and A. Sadri. The partitioned element method in computational solid mechanics. Comp. Meth. Appl. Mech. Engrg., 237-240:152-165, 2012.

88. M. Lin, J. Wang, Y. Wang, and X. Ye. Interior penalty discontinuous Galerkin method on very general polygonal and polyhedral meshes. 2012. Preprint available at http://arxiv.org/abs/1210.4214.

89. M. Lin, J. Wang, and X. Ye. Weak Galerkin finite element methods on polytopal meshes. 2012. Preprint available at http://arxiv.org/abs/1204.3655.

90. M. Lin, J. Wang, and X. Ye. Weak Galerkin finite element methods for the biharmonic equation on polytopal meshes. 2013. Preprint available at http://arxiv.org/abs/1303.0927.

91. K. Lipnikov, G. Manzini, and M. Shashkov. Mimetic finite difference method. J. Comput. Phys., 2013. accepted.

92. M. Shashkov. Conservative Finite Difference Methods. CRC Press, Boca Raton, FL, 1996.

93. M. Shashkov and S. Steinberg. Support-operator finite-difference algorithms for general elliptic problems. J. Comput. Phys., 118:131-151, 1995.

94. M. Shashkov and S. Steinberg. Solving diffusion equations with rough coefficients in rough grids. J. 
Comput. Phys., 129:383-405, 1996.

95. J. Hyman, M. Shashkov, and S. Steinberg. The numerical solution of diffusion problems in strongly heterogeneous non-isotropic materials. J. Comput. Phys., 132(1):130-148, 1997.

96. J.M. Hyman, M. Shashkov, and S. Steinberg. The effect of inner products for discrete vector fields on the accuracy of mimetic finite difference methods. Comput. Math. Appl., 42:1527-1547, 2001.

97. K. Lipnikov, M. Shashkov, and D. Svyatskiy. The mimetic finite difference discretization of diffusion problem on unstructured polyhedral meshes. J. Comput. Phys., 211(2):473-491, 2006.

98. Y. Kuznetsov, K. Lipnikov, and M. Shashkov. The mimetic finite difference method on polygonal meshes for diffusion-type problems. Comput. Geosci., 8:301-324, 2004.

99. K. Lipnikov, J. Morel, and M. Shashkov. Mimetic finite difference methods for diffusion equations on non-orthogonal non-conformal meshes. J. Comput. Phys., 199(2):589-597, 2004.

100. F. Brezzi, K. Lipnikov, and V. Simoncini. A family of mimetic finite difference methods on polygonal and polyhedral meshes. Math. Models Methods Appl. Sci., 15(10):1533-1551, 2005.

101. F. Brezzi, K. Lipnikov, and M. Shashkov. Convergence of mimetic finite difference method for diffusion problems on polyhedral meshes with curved faces. Math. Models Methods Appl. Sci., 16(2):275-297, 2006.

102. F. Brezzi, K. Lipnikov, M Shashkov, and V. Simoncini. A new discretization methodology for diffusion problems on generalized polyhedral meshes. Comput. Methods Appl. Mech. Engrg., 196(37-40):36823692, 2007.

103. K. Lipnikov, M. Shashkov, and I. Yotov. Local flux mimetic finite difference methods. Numer. Math., 112(1):115-152, 2009.

104. J.M. Hyman and M. Shashkov. The orthogonal decomposition theorems for mimetic finite difference methods. SIAM J. Numer. Anal., 36(3):788-818, 1999.

105. J. Hyman and M. Shashkov. Mimetic discretizations for Maxwell's equations and the equations of magnetic diffusion. Progress in Electromagnetic Research, 32:89-121, 2001.

106. K. Lipnikov, G. Manzini, F. Brezzi, and A. Buffa. The mimetic finite difference method for 3D magnetostatics fields problems on polyhedral meshes. J. Comput. Phys., 230(2):305-328, 2011.

107. F. Brezzi, A. Buffa, and G. Manzini. Mimetic scalar products for discrete differential forms, 2012. J. Comput. Phys., submitted.

108. K. Lipnikov, E. Nelson, and J. Reynolds. Mimetic discretization of two-dimensional magnetic diffusion equations. J. Comput. Phys., 2013. in press.

109. L. Margolin, M. Shashkov, and P. Smolarkiewicz. A discrete operator calculus for finite difference approximations. Comput. Methods Appl. Mech. Engrg., 187(3-4):365-383, 2000.

110. J. Campbell and M. Shashkov. A tensor artificial viscosity using a mimetic finite difference algorithm. J. Comput. Phys., 172(2):739-765, 2001.

111. K. Lipnikov and M. Shashkov. A framework for developing a mimetic tensor artificial viscosity for Lagrangian hydrocodes on arbitrary polygonal meshes. J. Comput. Phys., 229:7911-7941, 2010.

112. L. Beirão da Veiga, K. Lipnikov, and G. Manzini. Arbitrary-order nodal mimetic discretizations of elliptic problems on polygonal meshes. SIAM J. Numer. Anal., 49:1737-1760, 2011.

113. F. Brezzi, A. Buffa, and K. Lipnikov. Mimetic finite differences for elliptic problems. M2AN Math. Model. Numer. Anal., 43(2):277-295, 2009.

114. A. Cangiani, G. Manzini, and A. Russo. Convergence analysis of the mimetic finite difference method for elliptic problems. SIAM J. Numer. Anal., 47(4):2612-2637, 2009.

115. L. Beirão da Veiga, J. Droniou, and G. Manzini. A unified approach to handle convection terms in mixed and hybrid finite volumes and mimetic finite difference methods. IMA J. Numer. Anal., 31(4), 2011.

116. L. Beirão da Veiga, V. Gyrya, K. Lipnikov, and G. Manzini. Mimetic finite difference method for the Stokes problem on polygonal meshes. J. Comput. Phys., 228(19):7215-7232, 2009.

117. L. Beirão da Veiga, K. Lipnikov, and G. Manzini. Convergence of the mimetic finite difference method for the Stokes problem on polyhedral meshes. SIAM J. Numer. Anal., 48(4):1419-1443, 2010. 
118. L. Beirão da Veiga and K. Lipnikov. A mimetic discretization of the Stokes problem with selected edge bubbles. SIAM J. Sci. Comp., 32(2):875-893, 2010.

119. K. Lipnikov, D. Vassilev, and I. Yotov. Discontinuous Galerkin and mimetic finite difference methods for coupled Stokes-Darcy flows on polygonal and polyhedral grids. Numer. Math., 2013. accepted.

120. L. Beirão da Veiga. A mimetic finite difference method for linear elasticity. M2AN Math. Model. Numer. Anal., 44(2):231-250, 2010.

121. L. Beirão da Veiga. Finite element methods for a modified Reissner-Mindlin free plate model. SIAM J. Numer. Anal., 42(4):1572-1591, 2004.

122. L. Beirão da Veiga and D. Mora. A mimetic discretization of the Reissner-Mindlin plate bending problem. Numer. Math., 117(3):425-462, 2011.

123. A. Cangiani, F. Gardini, and G. Manzini. Convergence of the mimetic finite difference method for eigenvalue problems in mixed form. Comp. Meth. Appl. Mech. Engrg., 200(9-12):1150-1160, 2011.

124. K. Lipnikov, J.D. Moulton, and D. Svyatskiy. A Multilevel Multiscale Mimetic $\left(\mathrm{M}^{3}\right) \operatorname{method}$ for two-phase flows in porous media. J. Comput. Phys., 227:6727-6753, 2008.

125. L. Beirão da Veiga. A residual based error estimator for the mimetic finite difference method. Numer. Math., 108(3):387-406, 2008.

126. L. Beirão da Veiga and G. Manzini. An a posteriori error estimator for the mimetic finite difference approximation of elliptic problems. Int. J. Numer. Methods Engrg., 76(11):1696-1723, 2008.

127. V. Gyrya and K. Lipnikov. High-order mimetic finite difference method for diffusion problems on polygonal meshes. J. Comput. Phys., 227(20):8841-8854, 2008.

128. L. Beirão da Veiga and G. Manzini. A higher-order formulation of the mimetic finite difference method. SIAM J. Sci. Comp., 31(1):732-760, 2008.

129. L. Beirão da Veiga, K. Lipnikov, and G. Manzini. Convergence analysis of the high-order mimetic finite difference method. Numer. Math., 113(3):325-356, 2009.

130. C. D. Meyer. Matrix Analysis and Applied Linear Algebra. SIAM Publications, Philadelphia, PA, 2000.

131. T. Belytschko, Y. Krongauz, D. Organ, M. Fleming, and P. Krysl. Meshless methods: An overview and recent developments. Comp. Meth. Appl. Mech. Engrg., 139:3-47, 1996.

132. S. E. Mousavi, H. Xiao, and N. Sukumar. Generalized Gaussian quadrature rules on arbitrary polygons. Int. J. Numer. Methods Engrg., 82(1):99-113, 2009.

133. Y. Krongauz and T. Belytschko. Consistent pseudo-derivatives in meshless methods. Comp. Meth. Appl. Mech. Engrg., 146(2-3):371-386, 1997.

134. J. S. Chen, C. T. Wu, S. Yoon, and Y. You. A stabilized conforming nodal integration for Galerkin meshfree methods. Int. J. Numer. Methods Engrg., 50:435-466, 2001.

135. G. R. Liu, K. Y. Dai, and T. T. Nguyen. A smoothed finite element for mechanics problems. Comp. Mech., 39:859-877, 2007.

136. K. Y Dai, G. R. Liu, and T. T. Nguyen. A n-sided polygonal smoothed finite element method (nSFEM) for solid mechanics. Finite Elements in Analysis and Design, 43:847-860, 2007.

\section{Appendix A. A second-order accurate quadrature rule for polyhedral faces}

Let $f$ be a face of the polyhedral cell $\Omega_{e}, \mathbf{x}_{f}$ its barycenter, and $\mathbf{x}_{a}$ for $a=1, \ldots, n_{f}$ its vertices. We assume that these vertices are locally numbered by the sub-index $a$ so that their sequence forms the polygonal boundary of $f$. A second-order accurate quadrature rule using the vertices of $f$ as nodes requires a set of coefficients $\omega_{a}^{f}$ such that

$$
\mathbf{x}_{f}=\sum_{a=1}^{n_{f}} \omega_{a}^{f} \mathbf{x}_{a}, \quad \sum_{a=1}^{n_{f}} \omega_{a}^{f}=1, \quad \omega_{a}^{f}>0
$$


These weights are used to compute the entries of matrix $\mathbf{R}$ in (3.9b). We now proceed to show the existence of a set of weights that satisfies the properties in (A.1). The proof is constructive and leads to a formula for their calculation. At the end, we also discuss their non-uniqueness.

Let us first consider the case where $f$ is a convex polygon. If $f$ is a convex polygon, then the arithmetic average of the vertex positions

$$
\widehat{\mathbf{x}}_{f}=\frac{1}{n_{f}} \sum_{a=1}^{n_{f}} \mathbf{x}_{a}
$$

is an internal point of $f$. We decompose face $f$ in $n_{f}$ triangles obtained by connecting $\widehat{\mathbf{x}}_{f}$ and the vertices of $f$. In particular, let $T_{a}$ denote the triangle with vertices $\mathbf{x}_{a}, \mathbf{x}_{a+1}$ and $\mathbf{x}_{f}$, with the convention that $(\cdot)_{n_{f}+1}:=(\cdot)_{1}$ and $(\cdot)_{0}:=(\cdot)_{n_{f}}$. The barycenter of $T_{a}$ is given by

$$
\mathbf{x}_{T_{a}}=\frac{1}{\left|T_{a}\right|} \int_{T_{a}} \mathbf{x} d S=\frac{1}{3}\left(\mathbf{x}_{a}+\mathbf{x}_{a+1}+\widehat{\mathbf{x}}_{f}\right) .
$$

The barycenter of the polygonal face $f$ is the average of the barycenters of the triangles $T_{a}$ weighted by the area of the triangles. Indeed,

$$
\mathbf{x}_{f}=\frac{1}{|f|} \int_{f} \mathbf{x} d S=\frac{1}{|f|} \sum_{a=1}^{n_{f}} \int_{T_{a}} \mathbf{x} d S=\frac{1}{|f|} \sum_{a=1}^{n_{f}}\left|T_{a}\right| \mathbf{x}_{T_{a}} .
$$

Substituting (A.3) in (A.4), rearranging the summation indices, and noting that $|f|=\sum_{a=1}^{n_{f}}\left|T_{a}\right|$ and using (A.2) yields:

$$
\begin{aligned}
\mathbf{x}_{f} & =\frac{1}{3|f|} \sum_{a=1}^{n_{f}}\left|T_{a}\right|\left(\mathbf{x}_{a}+\mathbf{x}_{a+1}+\widehat{\mathbf{x}}_{f}\right)=\frac{1}{3|f|} \sum_{a=1}^{n_{f}}\left(\left|T_{a}\right|+\left|T_{a-1}\right|\right) \mathbf{x}_{a}+\frac{1}{3|f|} \widehat{\mathbf{x}}_{f} \sum_{a=1}^{n_{f}}\left|T_{a}\right| \\
& =\frac{1}{3|f|} \sum_{a=1}^{n_{f}}\left(\left|T_{a}\right|+\left|T_{a-1}\right|\right) \mathbf{x}_{a}+\frac{1}{3} \widehat{\mathbf{x}}_{f}=\frac{1}{3|f|} \sum_{a=1}^{n_{f}}\left(\left|T_{a}\right|+\left|T_{a-1}\right|+\frac{|f|}{n_{f}}\right) \mathbf{x}_{a} .
\end{aligned}
$$

Comparing (A.5) with (A.1) gives the formula for the weights:

$$
\omega_{a}^{f}=\frac{1}{3|f|}\left(\left|T_{a}\right|+\left|T_{a-1}\right|+\frac{|f|}{n_{f}}\right) .
$$

Clearly, it holds that $\omega_{a}^{f}>0$ for all index $a$. The first property in (A.1) is a consequence of the definition of the weights and (A.5); the second property can be checked by a direct calculation.

If face $f$ is nonconvex but star-shaped with respect to an internal point, then

$$
\widehat{\mathbf{x}}_{f}=\sum_{a=1}^{n_{f}} \beta_{a}^{f} \mathbf{x}_{a}
$$

for some choices of the coefficients $\beta_{a}^{f} \geq 0$ associated with the vertices $\mathbf{x}_{a}$. We can then repeat the previous argument (using the new expression of $\widehat{\mathbf{x}}_{f}$ ) and obtain the formula:

$$
\omega_{a}^{f}=\frac{1}{3|f|}\left(\left|T_{a}\right|+\left|T_{a-1}\right|+\beta_{a}^{f}|f|\right) .
$$

This formula can also be applied when face $f$ is convex. The simplest choice is $\beta_{a}^{f}=1 / n_{f}$, which returns (A.6). Alternative choices for $\beta_{a}^{f}$ gives a family of second-order accurate quadrature rules. 